\title{
An Algebraic Correspondence with Applications to Projective Bundles and Blowing up Chern Classes $\left(^{*}\right)$.
}

\author{
A. T. LASCU (Montreal) - D. B. ScotT (Brighton)
}

Dedicated to Professor Beniamino SEgRe

Summary. - New techniques are developed, based on the consideration of the projective bundle associated with a direct sum of two vector bundles, to give a simpler solution of the problem of blowing up Chern classes which was previously solved by Porteous [12] using the Grothendieck Riemann-Roch theorem.

\section{Dedication.}

It is a particular pleasure to the authors of this work to be allowed to dedicate it, on this happy jubilee, to Beniamino SEGRE. We deal with a problem which SEGRE and Topd brought to birth and to which SEgRe has made illuminating contributions over the years, and we hope that this paper will make the solution of the problem somewhat more accessible to classical algebraic geometers.

But we owe far more to SEGR than the vital seientific inspiration. It was he who brought us together and caused our paths to cross, and without him our collaboration wonld never have been undertaken. We both thank him trom the depths of our hearts for his deeplyvalued friendship, and for all he has done for both of us and for so many of our friends and colleagues in such widely separated lands.

\section{0. - Introduction.}

The problem of blowing up Chern classes is to compare the Chern classes of a variety $X$ with those of the variety $X^{\prime}$ obtained by blowing up $X$ along a subvariety $Y$. More precisely, if $f: X^{\prime} \rightarrow X$ is the "blowing down " morphism we have to calculate the difference between the total Chern class (or the Chern polynomial) of $X^{\prime}$ and the pull-back by $f$ of that of $X$.

This problem was first propounded (in terms of canonical systems) by Topd $[18,19,21]$ and further discussed by him in the essential survey article [22]. It was also discussed by SRGRE [17] and neatly reformulated by VAN DE VEN [23]. The first solution in general terms was given by PonTEous [12]: the proof depended on the Grothendieck Riemann-Roch Theorem, and was therefore fully effective only in characteristic zero. In positive characteristic it gave the result only modulo torsion. This latter reservation has now been implicitly removed by JovanoLoU [11]

(*) Entrata in Redazione il 2 aprile 1973. 
who has provided the necessary background results in positive characteristic. In this paper we give a more elementary solution of the problem, based on simple ideas of vector bundles and the geometry of their associated projective bundles. We develope the necessary techniques ab initio and try, in the interests of clarity and at some cost in brevity, to emphasise the geometry of the situation. In the course of this exposition we obtain a few well-known results; in some cases the proofs are essentially simpler than those generally known; in others our proofs are not more slick. But we have felt that it was worthwhile to develop our ideas systematically in a way that enables us to apply them as simply as possibly to our proof, in $\S 88$ and 9 , of the blowing up theorem.

The essential idea of our approach is that, since the Chern classes of an algebraic variety $X$ are derived (cf. GRoTHENDIECK [7]) from the geometry of its tangent direction bundle $\hat{X}$, we need to consider the "lifted correspondence" $\hat{f}$ induced between $\hat{X}^{\prime}$ and $\hat{X}$. The treatment of this is a development of the ideas put forward for correspondences between surfaces in [15]. One important point is that since $f$ is a birational morphism its graph is isomorphic with $X^{\prime}$. However $\hat{f}$ is not a morphism, and its graph is isomorphie with $\hat{X}^{\prime}$ blown up along a subvariety.

The calculations involved in a straightforward approach to $\hat{f}$, though not totally impracticable, are extremely unpleasant and we evade much of the difficult manipulation by a technical trick which enables us to reduce the solution of the general blowing up problem to that of a special case. This is the case where we blow up the base space (or zero-section) in the projective completion $\bar{E}$ of a vector-bundle $\mathbf{E}$. In our $\S 8$ we give a succinct account of this special case which is based on the exposition of our earlier sections and is greatly facilitated by a technical finesse in the calculations. In the following $\S 9$ we deal with the more difficult problem of reducing the general case to the special one.

The main tool in all this is the geometry associated with the algebraic correspondence $\Phi$ from $P\left(\mathrm{E}_{1} \oplus \mathrm{E}_{2}\right)$ to $P\left(\mathrm{E}_{1}\right) \times_{W} P\left(\mathrm{E}_{2}\right)$ where $\mathrm{E}_{1}, \mathrm{E}_{2}$ are vector bundles over the same base space $W$. The geometry of this situation is developed in $\$ 4$. However the essential geometric problems are already present in the, by no means trivial, special case where $W$ is a point. For didactic reasons we have chosen to develop the special case in $\S 2$ and use the geometric insights thereby gained to sketch the more general situation, rather than to adopt the less illuminating process of directly establishing the general results.

It is perhaps worth noting that the idea of investigating the geometry of $P\left(\mathrm{E}_{1} \oplus \mathrm{E}_{2}\right)$ arose from the consideration (cf. [15]) of the special case where the base space is a product $U \times V$ with projections $p_{V}, p_{F}$ onto its components, and $\mathrm{E}_{1}, \mathrm{E}_{2}$ are the pulled-up tangent bundles $p_{\sigma}^{*} T(U)$ and $p_{\nabla}^{*} T(V)$. In fact this special case plays an essential role in our treatment of the general blowing up problem.

We have already suggested the contents of $\S 2,4,8$ and 9 . In $\$ \S 1$ and 3 we are mainly concerned with stating basic results in a form convenient for later applications. The results of $\S 5,6$ and 7 are not, as we have already emphasised, all new, but the systematic treatment and the geometrical methods we offer are original. 
In conclusion we have to express our thanks to many people and organisations who have helped this work along. Our debt to Professor SEGRE is already recognised. We are especially indebted to the Science Research Council of Great Britain who gave us the opportunity to work together for a full year in the University of Sussex and we would like to express our appreciation of their support of which this is not the only fruit. We are also indebted not only to the University of Sussex but also to the University of Montreal who gave us the opportunity to come together again and, inter alia, finalise the presentation of this work. Our Sussex colleague A. J. KNIGHT has helped greatly by drawing attention to obscurities in an earlier draft. We owe as very special debt to I. R. PoRTEous, who has an obvious proprietary interest in this problem, who read our earlier draft with very great eare and from whose discerning comments we have learnt a very great deal, even if he may feel that we have not shown this as clearly as we might have done. Without their assistance the presentation of this work would have been less clear, but we must emphasise that neither KNIGHT nor PoRteous is responsible for the shorteomings and obscurities that remain.

\section{1. - Vector bundles and projective bundles over algebraic varieties.}

All the algebraie varieties with which we are concerned are defined over a fixed algebraically closed field, are quasi-projective and non-singular. (The old-fashioned geometer who prefers projective varieties can proceed as if we were discussing for the most part only projective varieties. However, we do in one or two places have to treat the space of a vector-bundle as a variety (cf. Lemmas 3.1 to 3.3).)

If $V$ is an algebraic variety, $\mathcal{A}(V)$ is its ring of rational equivalence classes (Chow ring) graded by co-dimension. To every morphism $f: U \rightarrow V$ corresponds a ring homomorphism $f^{*}: \mathcal{A}(V) \rightarrow \mathfrak{A}(U)$, and when $f$ is proper (in particular whenever $U$ is projective) there is also a group homomorphism $f_{*}: \mathcal{A}(U) \rightarrow \mathcal{A}(V)$ for the additive groups of these rings. Whereas $f^{*}$ preserves co-dimensions, i.e. the gradings of the Chow rings, $f_{*}$ preserves dimensions. These operations are connected by the projection formula (cf. GROTHENDIECK [4] p. 4-08)

$$
f_{*} a . b=f_{*}\left(a . f^{*} b\right)
$$

where $a \in \mathcal{A}(U), b \in \mathcal{A}(\nabla)$.

We are concerned with algebraic vector bundles $\mathrm{E}, \mathrm{F}, \mathrm{G}$ etc., over our algebraic varieties. If we wish to consider, say, $\mathrm{E}$ as an algebraic variety, we shall write $E$. The trivial line bundle over $X$ (i.e. the product $X \times \mathbb{A}^{1}$ ) will be denoted by $1_{X}$, which must be distinguished carefully from the unit of $A(X)$ which we call $1_{x}$.

The dual of the vector bundle $\mathrm{E}$ will be denoted by $\mathrm{E}$.

We shall denote by $P(\mathrm{E})$ the projective bundle of $\mathrm{E}$ whose fibres are the projective spaces derived from the fibres of $\mathrm{E}$. Wo shall denote by $\pi_{\mathrm{E}}$ the fibre pro- 
jection $\pi_{\mathrm{E}}: \mathrm{E} \rightarrow X$, where $X$ is the base space of $\mathrm{E}$. We shall denote by $\varrho_{\mathrm{E}}$ the fibre projection $\varrho_{\mathrm{E}}: P(\mathrm{E}) \rightarrow X$.

If $\mathrm{E}$ is a bundle (not necessarily a vector-bundle) over $X$ and we have a morphism $f: X \rightarrow X$ then there is a "pull-back " bundle $f * \mathrm{E}$ over $Y$ (for definition see [8] p. 43). In particular we can consider the pull-back $\varrho_{\mathrm{E}}^{*} \mathrm{E}$ of the bundle $\mathrm{E}$ to $P(\mathbf{E})$. If $z$ is a point of $P(\mathbf{E})$ with $\varrho_{E} z=x$, the fibre of $\varrho_{\mathrm{E}}^{*} \mathrm{E}$ at $z$ is a copy of $\mathbf{E}_{x}$ and contains the one-dimensional vector subspace which corresponds to the line of $\mathrm{E}_{x}$ giving rise to the point $z$ in the fibre $P(\mathrm{E})_{x}$. The aggregate of these lines is a line bundle over $P(\mathrm{E})$ (a sub-bundle of $\varrho_{\mathrm{E}}^{*} \mathrm{E}$ ) called the tantological bundle $\check{\mathrm{L}}_{\mathrm{E}}$ of $P(\mathrm{E})$. The notation is that of GrotHendiecK [7] who denotes by $\mathrm{L}_{\mathrm{E}}$ the dual of the tautological bundle.

In the next section we are particularly interested in the case where the base space of the bundles under discussion is a point. If $\mathrm{E}$ is a vector bundle over a point, i.e. an affine space, the bundle $P(\mathrm{E})$ is the associated projective space of $\mathrm{E}$ and $\varrho_{\mathrm{E}}^{*} \mathrm{E}$ is simply the product $P(\mathrm{E}) \times \mathrm{E}$ regarded as a trivial bundle over $P(\mathrm{E})$. But note that $\check{L}_{\mathrm{k}}$ is not trivial (unless $\mathrm{E}$ is a line bundle) and $\mathrm{L}_{E}$ is the bundle associated with the divisor class of a prime section of the projective space $P(E)$.

\section{2. - Some basic, and fairly elementary, geometry.}

We consider our basic correspondence in the case where we have two vector bundles $\mathrm{E}_{1}$ and $\mathrm{E}_{2}$, the base space of each being the single point $w$. Thus $\mathrm{E}_{1}$ and $\mathrm{E}_{2}$ are affine spaces $\mathbb{A}^{m+1}$ and $\mathbb{A}^{n+1}$ respectively (*).

We shall denote by $G$ the affine space $\mathbb{A}^{m+n+2}=\mathrm{E}_{1} \oplus \mathrm{E}_{2}$ (or $\mathrm{E}_{1} \times \mathrm{E}_{2}$ or even $\left.\mathrm{E}_{1} \times{ }_{w} \mathrm{E}_{2}\right)$. The associated projective spaces $\mathbb{P}^{m}=P\left(\mathrm{E}_{1}\right), \mathbb{P}^{n}=P\left(\mathrm{E}_{2}\right), \mathbb{P}^{m+n+1}=P(\mathrm{G})=$ $=P\left(\mathrm{E}_{1} \oplus \mathrm{E}_{2}\right)$, will be denoted by $P_{1}, P_{2}, R$ respectively. The Segre product $\mathbb{P}^{m_{2} n}$ of $\mathbb{P}^{m}$ and $\mathbb{P}^{n}$ will be written as $P_{1} \times P_{2}$.

If $\left(a_{0}, \ldots, a_{m}\right)$ and $\left(b_{0}, \ldots, b_{n}\right)$ are co-ordinates of points $A$ and $B$ (other than the origins) in $\mathrm{E}_{1}, \mathrm{E}_{2}$ respectively, they are also homogeneous co-ordinates for the associated points $a, b$ in $P_{1}, P_{2}$. The set of all points in $\mathrm{E}_{1}$ giving rise to the point $a$ in $P_{1}$, together with the origin, is a line $l_{a}$ in $\mathbf{E}_{1}$, and $l_{b}$ in $\mathbf{E}_{\mathrm{z}}$ is defined similarly. The aggregate of all $a \times l_{a}$ in $P_{1} \times \mathbf{E}_{1}$ is the tautological bundle $\breve{\mathrm{L}}_{\mathrm{E}_{1}}$ over $P_{1}$.

The vector $\left(a_{0}, \ldots, a_{m}, b_{0}, \ldots, b_{n}\right)$ represents the point $A \times B$ in G. And unless both of $A$ and $B$ are the origins it also gives the homogeneous co-ordinates of a point of $R$. We note that $R$ contains two subspaces (those respectively for which $B$ or $A$ is the origin) which we can identify with $P_{1}$ and $P_{2}$ : specifically we identify the point $\left(a_{0}, \ldots, a_{m}, 0, \ldots, 0\right)$ of $R$ with the point $a$ of $P_{1}$. With these identifica-

$\left({ }^{*}\right)$ In this section it will be found convenient to take the ranks of our bundles $\mathrm{E}_{1}$ and $\mathrm{E}_{2}$ to be $m+1$ and $n+1$ respectively (for typographical convenience it is easier to avoid assigning indices to the ranks here). In all subsequent sections the rank of $\mathrm{E}_{\text {(or }} \mathrm{E}_{i}$ ) will be denoted by $r$ (or $r_{i}$ ). It is hoped the reasons for this will appear clearly to the reader later. 
tions $R$ is the join of $P_{1}$ and $P_{2}$. We define a rational mapping $\Phi: R \rightarrow P_{1} \times P_{2}$ in which all the points of $R$ with homogeneous co-ordinates

$$
\left(\lambda a_{0}, \lambda a_{1}, \ldots, \lambda a_{m}, \mu b_{0}, \mu b_{1}, \ldots, \mu b_{n}\right)
$$

are mapped by $\Phi$ to the point $a \times b$ of $P_{1} \times P_{2}$.

The geometrical description of $\Phi$ is as follows. A point $c$ of $R \backslash P_{1} \cup P_{2}$ lies on a unique transversal of $P_{1}, P_{2}$ entting them in $a, b$ respectively. Then $\Phi$ maps $c$ (and all other points on the transversal) to the point $a \times b$ of $P_{1} \times P_{2}$. Clearly $P_{1}$ (unless $n=0$ ) and $P_{2}$ (unless $m=0$ ) are both fundamental for $\Phi$. Every point $a$ of $P_{1}$ is blown up (unless $n=0$ in which case it is simply transformed) into $a \times P_{2}$, and the transform of either $P_{1}$ or $P_{2}$ by $\Phi$ is the whole space $P_{1} \times P_{2}$.

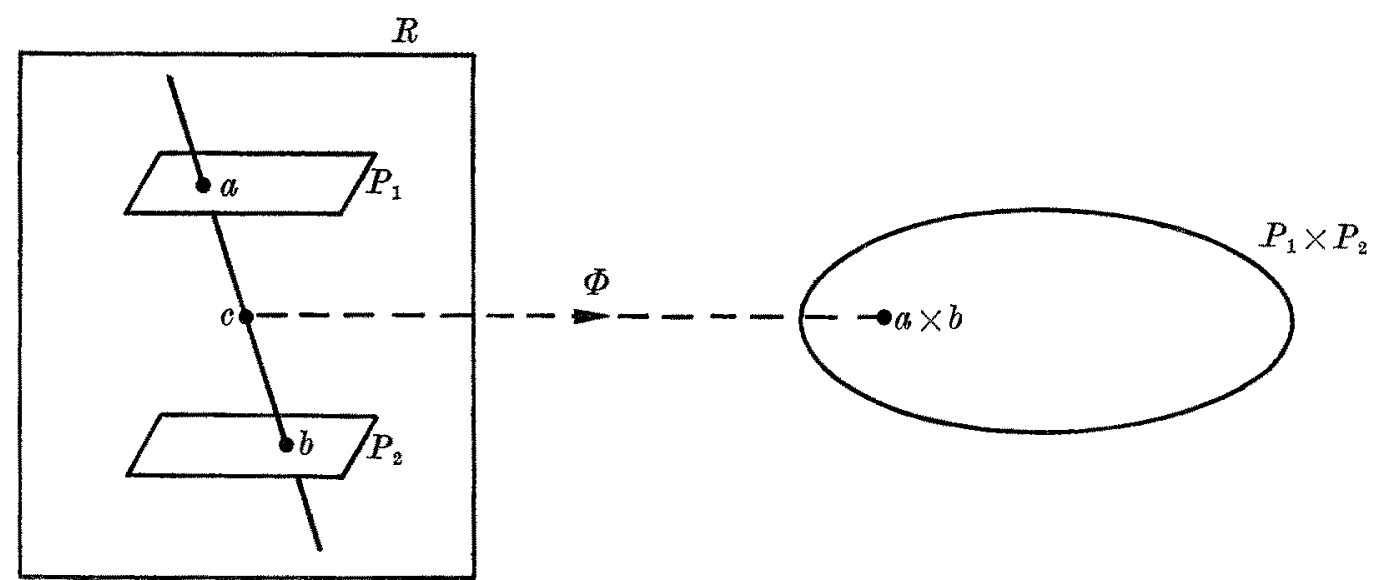

Fig. 2.1.

(In the above figure, and in subsequent figures and diagrams, we use a broken arrow to denote rational transformations, such as $\Phi$, which are not morphisms. This should produce no confusion with a similar convention, with rather different intent, introduced in the proof of lemma 3.2.)

Suppose now $m n>0$. Then we can blow up the fundamental subspaces $P_{1}$ and $P_{2}$ of $R$, thus transforming $R$ into a variety $H$ of the same dimension as $R$, and we shall denote by $\Psi$ the birational morphism $\Psi: H \rightarrow R$. Then $\Psi^{-1}\left(P_{1}\right)$ and $\Psi^{-1}\left(P_{2}\right)$ are both isomorphic with $P_{1} \times P_{2}$ (since the tangent lines to $R$, at a point $a$ of $P_{1}$, which do not touch $P_{1}$ are the joins of $a$ to $P_{2}$ ). Note that if we blow up by cubic primals of $R$ through $P_{1}$ and $P_{2}$ (which is possible because, since $m n \neq 0$, neither $P_{1}$ nor $P_{2}$ is a primal of $R$ ) the transversals of $P_{1}$ and $P_{2}$ will be transformed by $\Psi^{-1}$ into lines.

Suppose now $m n=0$. If both $m=0, n=0, \Phi$ is simply the mapping of a projective line to a point: there are no fundamental varieties on $R$ and we can take $H=R$. Less trivial is the case $m \neq 0, n=0$. In this case only the point $P_{2}$ is blown up and we can get the variety $H$ by transforming $R$ by quadrics through $P_{3}$ 
(in fact the cubics through $P_{1}$ and $P_{2}$ break up into $P_{1}$ and a quadrie through $P_{2}$ ) and again the transversals are transformed by $\Psi^{-1}$ into lines.

So in every case $H$ is a bundle of projective lines with two sections $\Psi^{-1}\left(P_{1}\right)$ and $\Psi^{-1}\left(P_{2}\right)$ which are each isomorphic with $P_{1} \times P_{2}$. In fact $H$ is obtained from $R$ by "separating " the transversals of $P_{1}$ and $P_{2}$ at their possible intersections on $P_{1}$ and $P_{2}$. As $H$ is a bundle of projective lines over $P_{1} \times P_{\mathrm{a}}$ it must be the projective bundle of a vector bundle of rank 2 over $P_{1} \times P_{2}$. We now show how this happens.

In the product $\left(P_{1} \times P_{2}\right) \times\left(\mathrm{E}_{1} \oplus \mathrm{E}_{2}\right)$ we consider the vector sub-bundle $S$ of rank 2 over $P_{1} \times P_{2}$ which is the aggregate of all points $(a \times b) \times\left(l_{a} \oplus l_{b}\right)$ (in more technical terms $S=p_{1}^{*} \check{\mathrm{L}}_{\mathrm{E}_{1}} \oplus p_{2}^{*} \breve{\mathrm{L}}_{\mathrm{E}_{2}}$, where $p_{1}, p_{2}$ are the projections from $P_{1} \times P_{2}$ to its components). It is easily checked that there is a birational morphism of $P(\mathbb{S})$ onto $R$ (for $P(\mathrm{~S})$ is a subspace of $\left(P_{1} \times P_{2}\right) \times R$ and we need only consider the projection of $P(S)$ onto the component $R$ of this ambient) for the inverse of which $P_{1}$ and $P_{2}$ are fundamental. So we verify that $P(\mathrm{~S})$ is actually isomorphic with $H$. So we have the following figure 2.2 , where $\Psi$ is a birational morphism, $\varrho_{\mathrm{s}}$ the projection of the projective bundle $H=P(\mathrm{~S})$, and $\Phi$ a rational mapping. We denote by $s_{1}$ and $s_{2}$ the isomorphisms of $P_{1} \times P_{2}$ with $\Psi^{-1}\left(P_{1}\right)$ and $\Psi^{-1}\left(P_{2}\right)$ respectively $\left({ }^{*}\right)$.

It is easily checked that $s_{1}\left(P_{1} \times P_{2}\right)$ arises from the points $(a \times b) \times\left(l_{a} \oplus 0\right)$ of $S$ so that $s_{1}\left(P_{1} \times P_{2}\right)=P\left(p_{1}^{*} \check{\mathrm{L}}_{\mathrm{F}_{2}}\right)$, but of course the projective bundle of any line bundle is isomorphic with the base space of the line-bundle. As the bundle $S$ has the two sub-line-bundles $p_{1}^{*} \breve{\mathrm{L}}_{\mathrm{E}_{1}} \oplus 0$ and $0 \oplus p_{2}^{*} \check{\mathrm{L}}_{\mathrm{E}_{2}}$ we naturally get the two sections in this way.

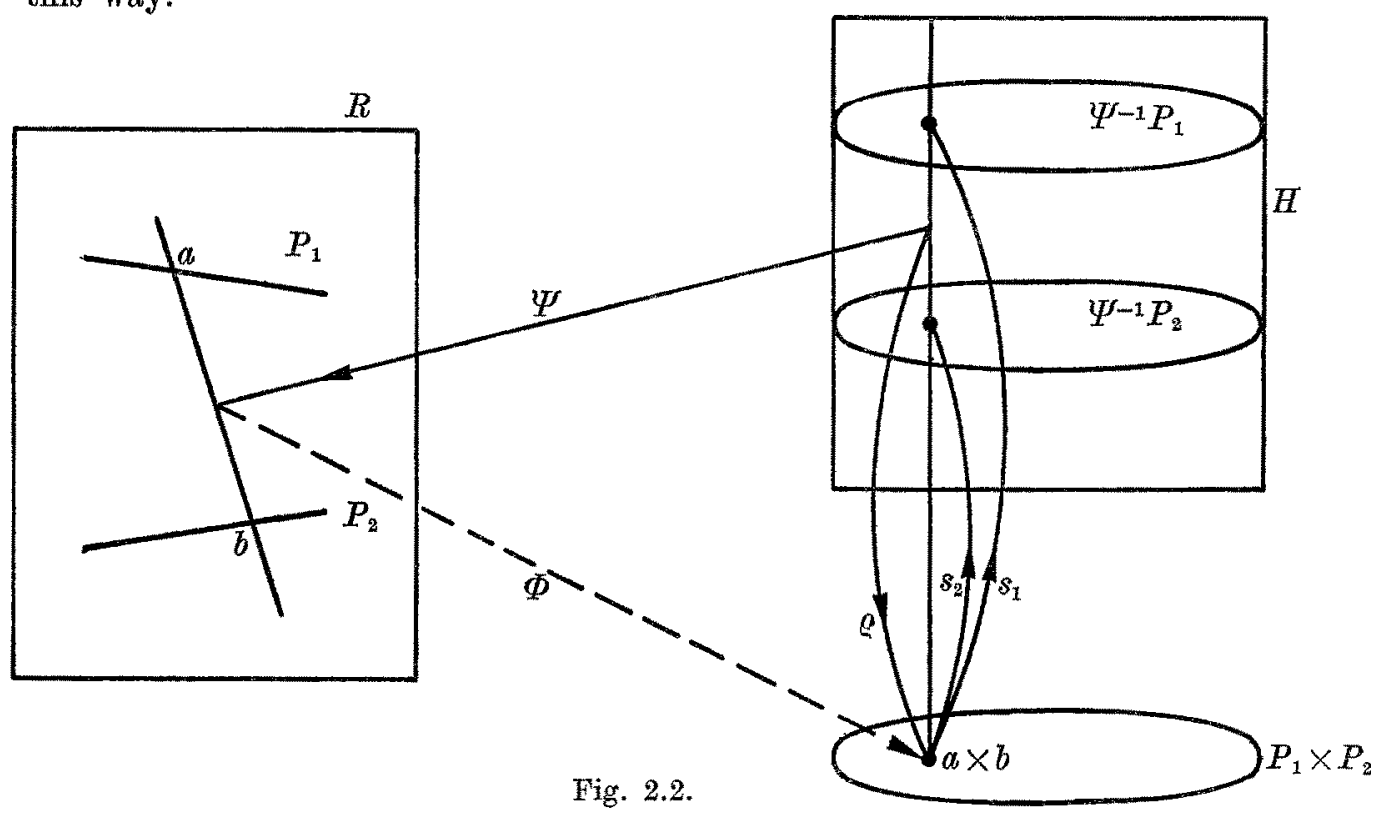

(*) NB. - It is an "abuse of language " to describe $s_{1}$ and $s_{2}$ as "sections " of the bundle $H$. If $i_{1}, i_{2}$ are the inclusions $i_{\alpha}: \Psi^{-1}\left(P_{\alpha}\right) \rightarrow H \quad(\alpha=1,2)$ it is $i_{1} s_{1}$ and $i_{2} s_{2}$ which are properly described as "sections". 
The case $n=0$ is worth a little thought. We can then identify $P_{1} \times P_{2}$ with $P_{1}$ : The mapping $\Phi$ is then essentially the projection of $R$ from the point $P_{2}$ onto the prime $P_{1}$. Any point of $R \backslash P_{1}$ can now be written uniquely in the form $\left(a_{0}, a_{1}, \ldots, a_{m}, 1\right)$ and this maps naturally onto the point $\left(a_{0}, \ldots, a_{m}\right)$ of $\mathrm{E}_{1}=\mathbb{A}^{m+1}$. Thus $R=\mathbb{P}^{m+1}$ is simply the "projective completion " of $\mathbb{A}^{m+1}$ by the "prime at infinity" $P_{1}$. In the same way $H$ is the projective completion of the dual of the tautological bundle of $P_{1}$ with $\Psi^{-1}\left(P_{2}\right)$ the "section at infinity" and $\Psi^{-1}\left(P_{1}\right)$ the zero section.

\section{3. - Tangent and normal bundles. Chern, Segre and Todd classes.}

The bundle of tangent vectors of a variety $X$ will be denoted by $\mathrm{T}(X)$ or $\mathrm{T}_{X}$. We shall frequently denote the bundle $P\left(\mathrm{~T}_{x}\right)$, the tangent direction bundle of $X$, by $\hat{X}$. We shall write simply $\varrho_{x}$ instead of $\varrho_{\mathrm{T}(X)}$ or $\varrho_{\mathrm{T}_{X}}$.

If $Y$ is a subvariety of $X$ and $i: Y \hookrightarrow X$ the inclusion, we have a pull-back $i^{*} T(X)$ of the tangent bundle of $X$ to $Y$. This is the bundle of tangent vectors to $X$ at points of $Y$ and has as a sub-bundle the bundle of tangent vectors to $Y$. The normal bundle $\mathrm{N}(Y, X)$ of $Y$ in $X$ is defined to be the quotient bundle: i.e. We have the exact sequence

$$
0 \rightarrow \mathrm{T}(Y) \rightarrow i^{*} \mathrm{~T}(X) \rightarrow \mathrm{N}(Y, X) \rightarrow 0
$$

of bundles over $Y$. In algebraic geometry there is no reason for assuming that the sequence (3.1) splits. There are however special cases of interest when it does.

We shall need later the following three lemmas which deal with the tangent bundle of a vector-bundle and the normal bundle of a sub-bundle.

Lewora 3.1. - Suppose $\mathbf{E}$ is a vector bundle over $X$. Then there is an exact sequence

$$
0 \rightarrow \pi_{\mathrm{E}}^{*} \mathrm{E} \rightarrow \mathrm{T}(E) \rightarrow \pi_{\mathrm{E}}^{*} \mathrm{~T}(X) \rightarrow 0 .
$$

The mapping $\mathrm{T}(E) \rightarrow \pi_{\mathrm{E}}^{*} \mathrm{~T}(X)$ derives from the tangent map of the projection $\pi_{\mathrm{E}}$ (cf. BouRbaKI [3], § 8.1.3).

The inclusion mapping of $\pi_{\mathrm{E}}^{*} \mathrm{E}$ in $\mathrm{T}(E)$ arises as follows. Let $u \in E$ and $x=\pi_{\mathrm{E}} u$. Then $\mathrm{E}_{x}$ is an affine space so that

$$
\left(\pi_{\mathrm{E}}^{*} \mathrm{E}\right)_{u}=\mathrm{E}_{x} \simeq \mathbf{T}\left(E_{x}\right)_{u}
$$

But the inclusion of $\mathrm{E}_{x}$ in $E$ thus gives rise to the inclusion of $\mathrm{T}\left(E_{x}\right)_{u}$ into $\mathrm{T}(E)_{u}$, i.e. an inclusion of $\pi_{\mathrm{E}}^{*} \mathrm{E}$ into $\mathrm{T}(E)$. To complete the proof we only need establish exactness and this is easily done for the special case where $\mathrm{E}=X \times \mathbb{A}^{*}$ in which case the sequence is easily seen to split. 
What this in fact shows is that $\pi_{\mathbb{E}}^{*} \mathrm{E}$ is the bundle of tangent vectors along the fibres of $E$ (cf. BourBakr loc. cit. and also the Example 5.5 of this paper where we consider the more difficult bundle of tangents along the fibres of a projective bundle).

LEMra 3.2. - Suppose $Y$ is a subvariety of $X$ with inclusion $i: Y \hookrightarrow X, \mathrm{E}$ a bundle over $X$ and $\mathrm{F}$ a sub-bundle of $i * \mathrm{E}$.

Then $0 \rightarrow \pi_{\mathrm{F}}^{*}\left(i^{*} \mathrm{E} / \mathrm{F}\right) \rightarrow \mathrm{N}(F, E) \rightarrow \pi_{\mathrm{F}}^{*} \mathrm{~N}(Y, X) \rightarrow 0$.

If $j$ is the inclusion $j: F \rightarrow E$ we have the commutative diagram

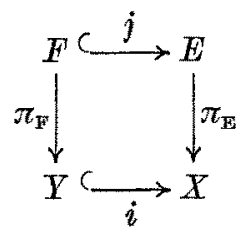

from which we deduce $\pi_{E} j=i \pi_{F}$ and hence

$$
j^{*} \pi_{\mathrm{E}}^{*}=\pi_{\mathrm{F}}^{*} i^{*}
$$

We then get the commutative diagram which follows $\left(^{*}\right)$, the first row is obtained by applying lemma 3.1 to $F$, the second applies the same lemma to $\mathrm{E}$ after which we apply $j^{*}$ and use 3.2 .

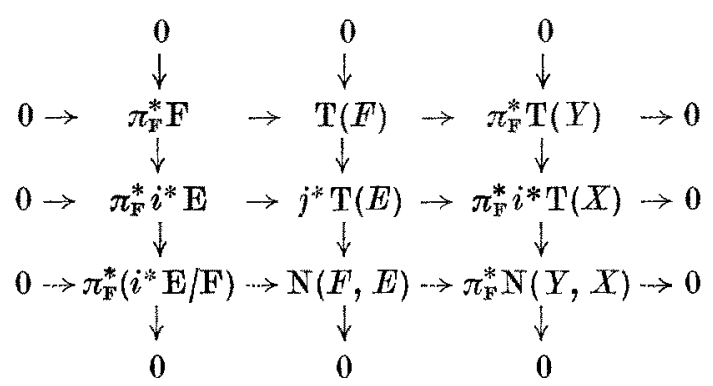

We now state as a lemma some special cases of Lemma 3.2.

$$
\begin{aligned}
& \text { Lemaia 3.3. - (a) } \mathrm{N}\left(i^{*} E, E\right) \simeq \pi_{i^{*} \mathrm{~N}}^{*} \mathrm{~N}(Y, X), \\
& \text { (b) If } \mathrm{E}, \mathrm{F} \text { are both bundles over } X, \mathrm{~N}(F, E) \simeq \pi_{\mathrm{F}}^{*}(\mathrm{E} / \mathrm{F}), \\
& \text { (c) } \mathrm{N}(X, E)=\mathrm{E} \text {. }
\end{aligned}
$$

To get $(a)$ we take $\mathbf{F}=i^{*} \mathrm{E}$. To get $(b)$ we take $Y=X$, to get (c) we take, in $(b), F$ as the zero bundle, so that the space of $\mathrm{F}$ is $X$ and $\pi_{F}$ is the identity.

(*) We adopt henceforth the convention that in any $3 \times 3$ diagram of short exact sequences we shall depict with dotted arrows the row or column whose exactness is being deduced from the remainder of the diagram. 
The normal bundle is vital in problems of blowing up subvarieties of a variety. Suppose $i: Y \hookrightarrow X$ is an inclusion and $X^{\prime}$ is a birational transform of $X$ obtained by "blowing up " $Y$ to a primal $Y^{\prime}$ in $X^{\prime}$ (so the codimension of $Y$ in $X$ is at least 2). If $f$ is the morphism $X^{\prime} \rightarrow X$ we have the commutative "blowing up " diagram below.

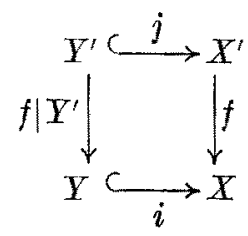

The variety $Y^{\prime}$ is a projective fibre bundle over $Y$. The essential features of this situation are summed up in the following two lemmas.

LEMMA 3.4. $-Y^{\prime}=P(\mathrm{E})$, where $\mathrm{E}=\mathrm{N}(Y, X)$.

LFMMA 3.5. $-\mathrm{N}\left(\bar{Y}^{\prime}, X^{\prime}\right)=\check{\mathrm{L}}_{\mathrm{E}}$.

The first result is obviously plausible, the second is less obvious. They are clearly implied in $[2, \S 12]$ and explicitly stated in $[12$, p. 123]. Note that the two lemmas above are unaffected if some subvariety of $X$ disjoint from $Y$ is also blown up, since the normal bundles are local properties.

For any vector bundle $\mathrm{E}$ over $V, C(t, \mathrm{E})$ is the Chern polynomial of $\mathrm{E}$, i.e. $C(t, \mathrm{E})=\sum_{i=0}^{r} c_{i} t^{i}$, where $r$ is the rank of $\mathrm{E}$ and $c_{i}=c_{i}(\mathrm{E})$, the $i$-th Chern class of $\mathrm{E}$, is an element of codimension $i$ in $\mathcal{A}(V)$.

The total Chern class $c(E)$ of $E$ is defined by

$$
c(\mathrm{E})=C(1, \mathrm{E})=c_{0}+\epsilon_{1}+\ldots+c_{r} .
$$

The Chern polynomial $C(t, X)$ of a variety $X$ (and the $i$-th Chern class of $X$ ) is defined by

$$
C(t, X)=C\left(t, \mathrm{~T}_{X}\right)
$$

We can also consider the Segre classes $s_{i}(E)$, or inverse Chern classes, of $\mathrm{E}$ (or $X$ ) by inverting in $\mathcal{A}(X)[t]$ the Chern polynomials to give Segre polynomials $S(t, \mathbf{E})$ where

$$
O(t, \mathbf{E}) . S(t, \mathbf{E})=1
$$

These classes have been introduced independently by TODD and SEGRE: initially introduced for manipulative convenience, they were given a significance by SEGRE [17] in line with our requirements here. 
The essential properties of Chern classes are the following. If $f: Y \rightarrow X$ is a morphism and $\mathrm{E}$ is a bundle over $X$ then

$$
f^{*} c_{i}(\mathbf{E})=e_{i}\left(f^{*} \mathbf{E}\right)
$$

and if $0 \rightarrow \mathrm{E} \rightarrow \mathrm{F} \rightarrow \mathrm{G} \rightarrow 0$ is a short exact sequence of bundles over $X$ then

$$
O(t, \mathrm{~F})=O(t, \mathrm{E}) C(t, \mathrm{G})
$$

We shall denote by $\bar{C}(t, \mathrm{E})$ the reversed Chern polynomial of $\mathrm{E}$ defined by

$$
\bar{C}(t, \mathrm{E})=t^{r} C\left(t^{-1}, \mathrm{E}\right)=t^{r} c_{0}(\mathrm{E})+t^{r-1} e_{1}(\mathrm{E})+\ldots+e_{r}(\mathrm{E}),
$$

$r$ again being the rank of $\mathrm{E}$.

If $Y$ is a subvariety of $X$ of eo-dimension $r$ we shall describe as the Todd polynomial $T(t ; Y, X)$ and the Todd classes of immersion $\left(^{*}\right) t_{i}(Y, X)(i=0,1, \ldots, r)$ the Ohern polynomial and the Chern classes of the normal bundle of $Y$ in $X$. The total Todd class $t(Y, X)$ is defined in the obvious way. The Todd classes are in $\mathcal{A}(Y)$.

If $Y$ is a primal of $X$, a subvariety of co-dimension 1 , and $i: Y \rightarrow X$ is the inclusion mapping, it is known (and easily proved) that

$$
t_{1}(Y, X)=i^{*} i_{*}\left(1_{Y}\right)
$$

the result being equivalent, in elassical terminology, to the adjunction formula for canonical primals.

If $\mathbf{E}$ is a vector bundle (of rank $r$ ) on $V$ we have on $P(\mathbf{E})$ the Grothendieck class $\xi_{\mathrm{E}}$ (if $\mathrm{E}=\mathrm{T}(V)$ we may write $\xi_{V}$ instead of $\xi_{E}$ ), an element of co-dimension 1 in $\mathcal{A}(P(\mathrm{E})$ ): it is defined by

$$
\xi_{\mathrm{E}}=c_{1}\left(\mathrm{~L}_{\mathrm{k}}\right)=-c_{1}\left(\check{\mathrm{L}}_{\mathrm{E}}\right)
$$

The ring $\mathfrak{A}(P(\mathrm{E}))$ is a finitely generated module over its subring $\varrho^{*} \mathfrak{A}(V)$ (an isomorphic image of $\mathcal{A}(V))$ with basis $\left(1, \xi_{\mathrm{E}}, \ldots, \xi_{\mathrm{m}}^{r-1}\right)$. The class $\xi_{\mathrm{F}}$ satisfies the minimal equation (over $\varrho^{*} \mathcal{A}(V)$ )

$$
\varrho^{*} c_{0} \cdot \xi_{\mathrm{x}}^{r}+\varrho^{*} e_{1} \cdot \xi_{\mathrm{z}}^{r-1}+\ldots+\varrho^{*} e_{r}=0 .
$$

We write this as

$$
\left[\varrho^{*} \bar{C}\right]\left(\xi_{\mathrm{E}}, \mathbf{E}\right)=\mathbf{0}
$$

$\left(^{*}\right)$ These classes were discovered by TODD [20] and rediscovered by SEGRE [16, 17] in different ways. The authors wonld like to describe as the Segre classes of immersion those derived from the inverse polynomial of $T$ (as the Segre classes are inverse to the Chern classes) but we have no need of them in this paper. 
where $\varrho^{*}$ is applied to the coeffieients of the polynomial $\bar{C}(t, \mathrm{E})$ and $\xi_{\mathrm{E}}$ is substituted for $t$ after the application of $e^{*}$.

Following GRoTHENDIEOK [7] we shall take (3.10) as the definition of the Chern classes.

Segre's original definition [17] of the Segre classes $s_{i}(\mathrm{E})$ is equivalent (cf. GaLBURĂ [5]) to the assertion that

$$
s_{i}(\mathrm{E})=\varrho_{\mathrm{E} *}\left(\xi_{\mathrm{E}}^{r-1+i}\right) .
$$

We conclude this section with some results on sub-bundles of bundles. Lemma 3.6 is trivial. Theorems 3.7 and 3.8 are basic results established in [9]. Theorem 3.7 is there proposition 1 of section 3 and Theorem 3.8 is the first lemma of the same section.

Lemara 3.6. - Suppose $\mathbf{E}$ is a vector bundle over $V$, that $i: U \hookrightarrow V$ is an inclusion mapping and that $\mathrm{F}$ is a sub-bundle of $i^{*} \mathrm{E}$. There is then an inclusion mapping $j: P(\mathrm{~F}) \hookrightarrow P(\mathrm{E})$ and

$$
j^{*} \check{\mathrm{L}}_{\mathrm{E}}=\check{\mathrm{L}}_{\mathrm{F}}, \quad j^{*} \xi_{\mathrm{E}}=\xi_{\mathrm{F}} .
$$

THEOREM 3.7 (Scott's formula) (*). - With the hypotheses of the preceding lemma, the equivalence class of $P(\mathrm{~F})$ in $P(\mathrm{E})$ is given by

$$
c l_{P(\mathrm{E})} P(\mathrm{~F})=j_{*}\left(1_{P(\mathrm{~F})}\right)=\left[Q_{E}^{*} i_{*} \bar{C}\right]\left(\xi_{\mathrm{E}}, i^{*} \mathrm{E} / \mathrm{F}\right) .
$$

THeOREM 3.8. - Suppose $\mathrm{E}$ is a vector bundle over $V$ of rank $r$ and $a$ is an element of $\mathcal{A}(P(E))$ so that, as remarked above, there is an unique expression $a=\sum_{i=1}^{r} \varrho^{*} a_{i} \cdot \xi_{\mathrm{E}}^{r-1}$, where $a_{i}$ is an element of $\mathfrak{A}(V)$ (not necessarily of co-dimension $i$ or even homogeneous) Then if $\bar{a}$ in $\mathcal{A}(\nabla)$ is defined by $\bar{a}=\sum_{i=1}^{\infty} a_{i}$, we have

$$
\bar{a}=\varrho_{\mathrm{E}} *\left(a \sum_{0}^{\infty} \xi_{\mathrm{E}}^{i}\right) \cdot \boldsymbol{c}(\mathrm{E})
$$

(Of course, the infinite sum is purely formal: $\xi_{\mathrm{E}}^{k}=0$ for $k \geqslant r+\operatorname{dim} V$.)

\section{4. - Some basic geometry associated with projective bundles.}

Suppose that $\mathrm{E}_{1}, \mathrm{E}_{2}$ are vector-bundles over an algebraic variety, which (for reasons which will appear) we shall now call $W$, and let $\mathrm{G}=\mathrm{E}_{1} \oplus \mathrm{E}_{2}$. Consider the

(*) The reason for the name of this formula, ehristened by the authors of [9] seems obscure to me. In [14] Scont only provided an incomplete proof of a very restricted case. 
projective bundle $P(\mathrm{G})$ and the bundle (of two-way projective spaces) $P\left(\mathrm{E}_{1}\right) \times_{W} P\left(\mathrm{E}_{2}\right)$ which, for brevity, we shall in future call $Z$. For any point $w$ of $W$ the relationship between the fibres $R=P(G)_{w}$ and $Z_{w}=P\left(\mathrm{E}_{1}\right)_{w} \times P\left(\mathrm{E}_{2}\right)_{w}=P_{1} \times P_{2}$ is precisely that discussed in $\S 2$. The results of that section globalise in a straightforward manner.

Thus $P(\mathrm{G})$ has two sub-bundles, identified with $P\left(\mathrm{E}_{1}\right)$ and $P\left(\mathrm{E}_{2}\right)$, of which it is the "join " (each fibre of $P(\mathrm{G})$ being the join of the corresponding fibres of $P\left(\mathrm{E}_{1}\right)$ and $\left.P\left(\mathrm{E}_{2}\right)\right)$. We can combine the maps $\Phi_{w}$, say, in the various fibres, to give a global rational transformation $\Phi$ from $P(\mathrm{G})$ to $Z$. The transformation $\Phi$ has $P\left(\mathrm{E}_{1}\right)$ as a fundamental locus unless $\mathrm{E}_{2}$ is a line-bundle and $P\left(\mathrm{E}_{2}\right)$ is a fundamental locus unless $\mathrm{E}_{1}$ is a line-bundle.

Just as in the fibres (cf. $\S 2$ ) we can blow up $P(\mathrm{G})$ along $P\left(\mathrm{E}_{1}\right)$ and $P\left(\mathrm{E}_{2}\right)$ to get a variety $H$ with a birational morphism $\Psi$ from $H$ to $P(\mathrm{G})$. Both $\Psi^{-1} P\left(\mathrm{E}_{1}\right)$ and $\Psi_{-1} P\left(\mathrm{E}_{2}\right)$ are isomorphic to $Z=P\left(\mathrm{E}_{1}\right) \times_{W} P\left(\mathrm{E}_{2}\right)$, so we shall call them respectively $Z_{1}$ and $Z_{2}$. The variety $H$ is a projective line-bundle over $Z$. If $q_{1}, q_{2}$ (we shall use $p_{1}, p_{2}$ for the projections from a product to its first and second components: we use $q_{1}, q_{2}$ because we have not a true product but a restricted one) are the projections from $Z=P\left(\mathrm{E}_{1}\right) \times_{W} P\left(\mathrm{E}_{2}\right)$ onto $P\left(\mathrm{E}_{1}\right)$ and $P\left(\mathrm{E}_{2}\right)$ respectively, then $H=P(\mathrm{~S})$ where $S=q_{1}^{*} \breve{\mathrm{L}}_{\mathrm{R}_{1}} \oplus q_{2}^{*} \breve{\mathrm{L}}_{\mathrm{E}_{2}}$. The sections $Z_{1}$ and $Z_{2}$ of $H$ are isomorphic with the bundles $P\left(q_{1}^{*} \check{\mathrm{L}}_{\mathrm{E}_{2}}\right)$ and $P\left(q_{2}^{*} \check{\mathrm{L}}_{\mathrm{E}_{2}}\right)$ respectively. Again we denote the isomorphic mappings of $Z$ onto $Z_{1}$ and $Z_{2}$ by $s_{1}$ and $s_{2}$ respectively and $i_{1}, i_{2}$ are the inclusion mappings of $Z_{1}, Z_{2}$ into $H$.

All this is summed up in fig. 4.1 below and the diagram $(4,2)$.

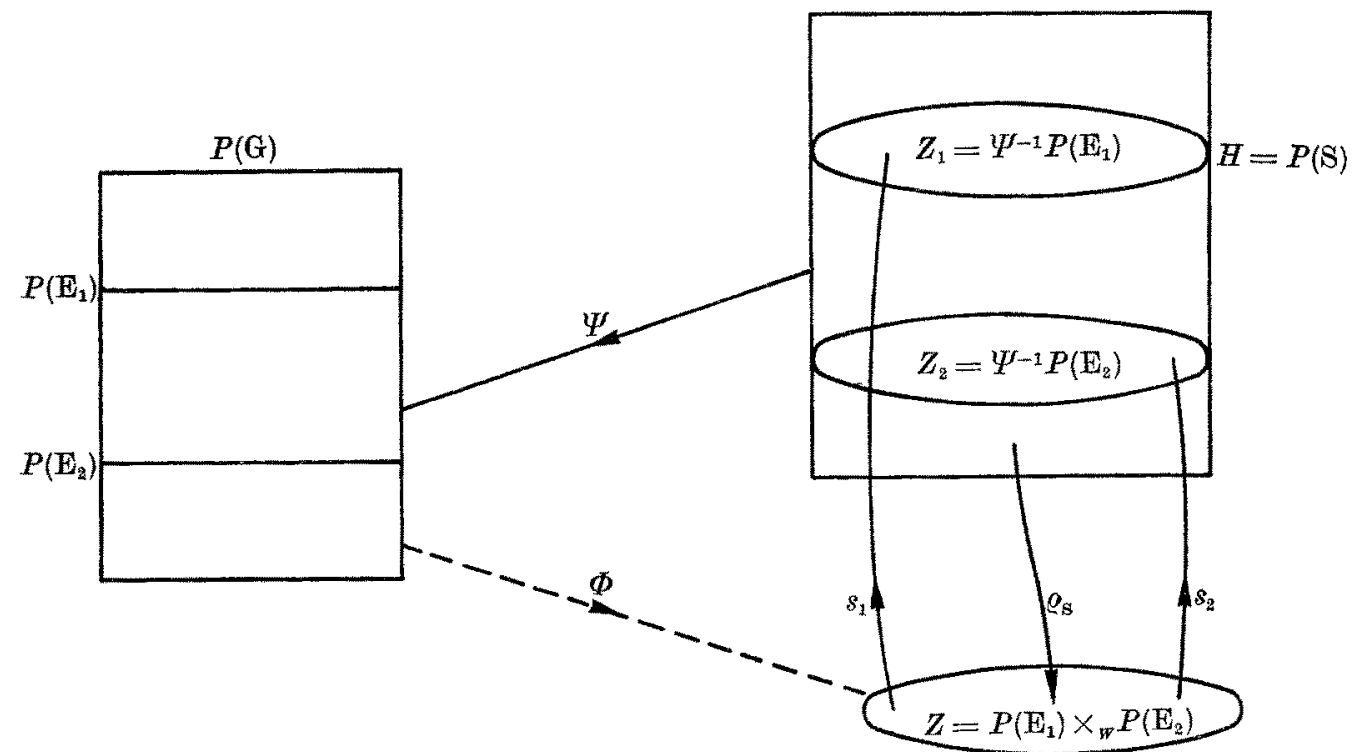

Fig. 4.1. 
In the diagram below we have omitted the projection $\varrho_{G}: P(\mathrm{G}) \rightarrow W$ and the inclusion maps of $P\left(\mathrm{E}_{1}\right)$ and $P\left(\mathrm{E}_{2}\right)$ in $P(\mathrm{G})$.

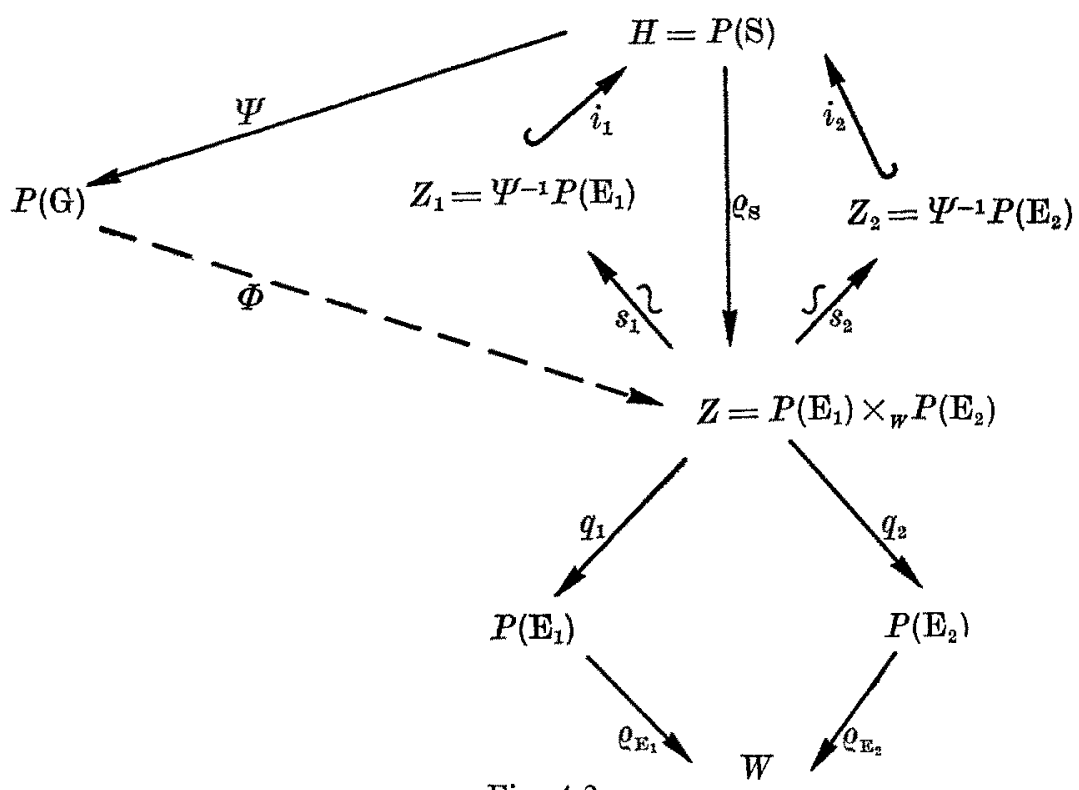

Fig. 4.2.

The situation in the fibres above any point of $W$ is precisely that depicted in fig. 2.2.

Consider now the various Grothendieck elasses $\xi_{\mathrm{s}}, \xi_{\mathrm{E}_{1}}, \xi_{\mathrm{E}_{\mathrm{g}}}$ in $\mathcal{A}(H), \mathcal{A}\left(P\left(\mathrm{E}_{1}\right)\right)$ and $A\left(P\left(\mathrm{E}_{2}\right)\right)$ respectively which are the first Chern classes (i.e. of codimension 1 ) of $\mathrm{L}_{\mathrm{s}}, \mathrm{L}_{\mathrm{E}_{1}}, \mathrm{~L}_{\mathrm{E}_{2}}$. To simplify our symbols a little we shall write

$$
\xi_{1}=q_{1}^{*} \xi_{\mathrm{E}_{1}}, \quad \xi_{2}=q_{2}^{*} \xi_{\mathrm{E}_{2}},
$$

these both being classes of $\mathcal{A}(Z)$. We shall also write

$$
\check{\mathrm{L}}_{1}=q_{1}^{*} \check{\mathrm{L}}_{\mathrm{E}_{1}}, \quad \check{\mathrm{L}}_{2}=q_{2}^{*} \check{\mathrm{L}}_{\mathrm{E}_{2}},
$$

these being bundles over $Z$. It is clear that

$$
\xi_{1}=-c_{1}\left(\check{\mathrm{L}}_{1}\right), \quad \xi_{2}=-c_{1}\left(\check{\mathrm{L}}_{2}\right) .
$$

We now find the equivalence classes of $Z_{1}$ and $Z_{2}$ in $H$ and also their Todd classes. These are given by the following

THEOREM 4.1. - (i) $\mathrm{cl}_{H} Z_{1}=i_{1}^{*}\left(1_{Z_{1}}\right)=\xi_{\mathrm{s}}-\varrho_{\mathrm{s}}^{*} \xi_{\mathrm{a}}$,

(ii) $\mathrm{cl}_{z} Z_{2}=i_{2}^{*}\left(1_{Z_{2}}\right)=\xi_{\mathrm{s}}-\varrho_{\mathrm{s}}^{*} \xi_{1}$,

(iii) $s_{1}^{*} t_{1}\left(Z_{1}, H\right)=\xi_{1}-\xi_{2}$,

(iv) $s_{2}^{*} t_{1}\left(Z_{2}, H\right)=\xi_{2}-\xi_{1}$.

2 - Annali di Matematica 
Olearly we need only establish (i) and (iii). To prove (i) we remark that $H=P(\mathrm{~S})=P\left(\check{\mathrm{L}}_{1} \oplus \check{\mathrm{L}}_{2}\right)$, and $Z_{1}$ is the variety of $H$ representing $P\left(\check{\mathrm{L}}_{1}\right)$. We can thus apply Theorem 3.7 where $i$ is now the identity and $\mathrm{E}, \mathrm{F}, i * \mathrm{E} / \mathrm{F}$ are to be replaced by $\mathrm{S}, \check{\mathrm{L}}_{1}, \check{\mathrm{L}}_{2}$.

So $\mathrm{cl}_{H}\left(Z_{1}\right)=i_{1 *}(1)=\left[\varrho_{\mathrm{s}}^{*} \bar{C}\right]\left(\xi_{\mathrm{s}}, \check{\mathrm{L}}_{2}\right)=\xi_{\mathrm{s}}-\varrho_{\mathrm{s}}^{*} \xi_{2}$.

To prove (iii) we remark that, by (3.7),

$$
s_{1}^{*} t_{1}\left(Z_{1}, H\right)=s_{1}^{*} i_{1}^{*}\left(i_{1 *}(1)\right)=s_{1}^{*} i_{1}^{*} \xi_{\mathrm{s}}-s_{1}^{*} i_{1}^{*} \varrho_{\mathrm{s}}^{*} \xi_{2} .
$$

But $\varrho_{\mathrm{s}} i_{1} s_{1}$ is the identity mapping on $Z$, so $s_{1}^{*} i_{1}^{*} \varrho_{\mathrm{s}}^{*} \xi_{2}=\xi_{2}$. Also $s_{1}^{*} i_{1}^{*} \xi_{\mathrm{s}}=$ $=-s_{1}^{*} i_{1}^{*} o_{1}\left(\check{\mathrm{L}}_{\mathrm{s}}\right)=-c_{1}\left(s_{1}^{*} i_{1}^{*} \breve{\mathrm{L}}_{\mathrm{s}}\right)$. But (lemma 3.6) $i_{1}^{*} \check{\mathrm{L}}_{\mathrm{s}}$ is the tantological bundle on $Z_{1}$ regarded as $P\left(\check{\mathrm{L}}_{1}\right)$. As $s_{1}$ is an isomorphism it follows that $s_{1}^{*} i_{1}^{*} \check{\mathrm{L}}_{\mathrm{s}}$ is the tautological bundle on $Z$ regarded as $P\left(\check{\mathrm{L}}_{1}\right)$, i.e. $s_{1}^{*} i_{1}^{*} \check{\mathrm{L}}_{\mathrm{s}}=\check{\mathrm{L}}_{1}$. So $-c_{1}\left(s_{1}^{*} i_{1}^{*} \check{\mathrm{L}}_{\mathrm{g}}\right)=\xi_{1}$ and the theorem is proved.

Next we calculate the classes of $P\left(\mathrm{E}_{1}\right)$ and $P\left(\mathrm{E}_{2}\right)$ in $P(\mathrm{G}) . \quad \mathrm{As} \mathrm{G}=\mathrm{E}_{1} \oplus \mathrm{E}_{2}$ a further application of Theorem 3.7 gives us

THEOREM 4.2. $-\mathrm{cl}_{P(\alpha)}\left(P\left(\mathrm{E}_{1}\right)\right)=\left[\varrho_{\theta}^{*} \bar{C}\right]\left(\xi_{G}, \mathrm{E}_{2}\right), \mathrm{cl}_{P(\alpha)}\left(P\left(\mathrm{E}_{2}\right)\right)=\left[\varrho_{\theta}^{*} \bar{C}\right]\left(\xi_{G}, \mathrm{E}_{1}\right)$.

The calculation of the Todd classes of $P\left(\mathrm{E}_{1}\right)$ and $P\left(\mathrm{E}_{2}\right)$ in $P(G)$ is harder than for their inverse images under $\Psi$, because in general we are not dealing with primals. The result will be obtained as Theorem 7.3.

Three special cases of all this are of interest later. The first is important in the theory of correspondences, and is useful in the final section of this paper. Suppose $W=U \times V$ and $p_{\sigma}, p_{V}$ are the projections $p_{1}, p_{2}$ to the components. Consider $\mathrm{E}_{1}=p_{v}^{*} \mathrm{~T}(U), \mathrm{E}_{2}=p_{V}^{*} \mathrm{~T}(V)$. Then $P\left(\mathrm{E}_{1}\right)=\hat{U} \times V, P\left(\mathrm{E}_{2}\right)=V \times \hat{V}$, and, of course. $P(\mathrm{G})=\widehat{U \times V}$. Theorem 4.2 in this case becomes

THEOREM 4.3. $-\mathrm{cl}_{\widehat{U \times V}}(\hat{O} \times V)=\left[\varrho_{U \times \gamma}^{*} p_{V}^{*} \bar{C}\right]\left(\xi_{U \times V}, V\right)$.

The second special case is where $\mathrm{E}_{1}=\mathrm{E}_{2}=\mathrm{E}$. In that case $Z=P\left(\mathrm{E}_{1}\right) \times_{W} P\left(\mathrm{E}_{2}\right)$ is isomorphic with $P\left(\varrho^{*} \mathrm{E}\right)=\varrho^{*} P(\mathrm{E})$, and we shall adopt the convention that $\varrho_{Q^{*} R}=q_{1}$. This is further discussed in example 5.4.

The third special case is where $\mathrm{E}_{2}=\mathbf{1}_{W}$, the trivial line bundle over $W$. A comparison with the concluding paragraph of $\S 2$ shows that in this case $P\left(\mathrm{E}_{1} \oplus 1\right)$ is the projective closure $\bar{E}_{1}$ of $\mathrm{E}_{1}$. Here the subvarieties $P\left(\mathrm{E}_{1}\right)$ and $P(1)$ (a copy of $W$ ) give respectively the locus of the primes at infinity in the fibres and the zero section respectively. A detailed treatment of this situation is given in $\$ 6$.

\section{5. - Tensor products with line-bundles. The bundle of tangents along the fibres of a projective bundle.}

Suppose we have two bundles $B_{1}$ and $B_{2}$ over a variety $W$ such that the projective bundles $P\left(\mathrm{~B}_{1}\right)$ and $P\left(\mathrm{~B}_{2}\right)$ are isomorphic. This means that we have a commutative 
diagram

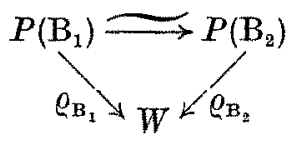

Then it is trivial that there is a line-bundle $L$ over $W$ such that $B_{1}=B_{2} \otimes L$.

Let us now identify $P\left(\mathrm{~B}_{1}\right)$ and $P\left(\mathrm{~B}_{2}\right)$ and denote both of them by $P(\mathrm{~B})$, and denote both the projections by $\varrho_{\mathrm{B}}$. Pulling back our bundles to $P(\mathrm{~B})$ gives

$$
\varrho_{\mathrm{B}}^{*} \mathrm{~B}_{1}=\varrho_{\mathrm{B}}^{*} \mathrm{~B}_{2} \otimes \varrho_{\mathrm{B}}^{*} \mathrm{~L} .
$$

It follows, by considering the tautological bundles, that

$$
\check{\mathrm{L}}_{\mathrm{B}_{1}}=\check{\mathrm{L}}_{\mathrm{B}_{\mathrm{z}}} \otimes \varrho_{\mathrm{B}}^{*} \mathrm{~L} \text {. }
$$

Thas $\varrho_{B}^{*} \mathrm{~L}$ is uniquely defined as a bundle over $P(B)$ and (because the divisor associated with a tensor product of line bundles is the sum of the divisors associated with the two bundles)

$$
\xi_{\mathrm{B}_{\mathrm{z}}}-\xi_{\mathrm{B}_{1}}=c_{1}\left(\check{\mathrm{L}}_{\mathrm{B}_{1}}\right)-c_{1}\left(\check{\mathrm{L}}_{\mathrm{B}_{\mathrm{g}}}\right)=c_{1}\left(\varrho_{\mathrm{B}}^{*} \mathrm{~L}\right)=\varrho_{\mathrm{B}}^{*} c_{1}(\mathrm{~L})
$$

But $\varrho_{\mathrm{B}}^{*}$ is an injection of $\mathfrak{A}(W)$ into $\mathcal{A}(P(B))$ so that $c_{1}(L)$ is uniquely defined, and thus $\mathrm{L}$ is defined to within isomorphism. We summarise all this as

Lavara 5.1. - If the bundles $\mathrm{B}_{1}$ and $\mathrm{B}_{2}$ have the same projective bundles over $W$ with projection $\varrho_{\mathrm{B}}$, there is a line-bundle $\mathrm{L}$ over $\mathrm{W}$ defined to within isomorphism such that $\mathrm{B}_{1}=\mathrm{B}_{2} \otimes \mathrm{L}$ and $\xi_{\mathrm{B}_{2}}-\xi_{\mathrm{B}_{1}}$ is the $\varrho_{\mathrm{B}}^{*}$ image of the first Chern class of $\mathrm{L}$.

A consequence of the usual rules for manipulating Chern classes $[8, p .64]$ is the following useful

Lewara 5.2. - If $\mathrm{L}$ is a line bundle and $\eta=c_{1}(\mathrm{~L})$ then

$$
\bar{C}(t, \mathbf{E} \otimes \mathrm{L})=\bar{C}(t+\eta, \mathbf{E}) .
$$

We give three examples of situations where different bundles have the same projective bundle.

ExampLe 5.3. - If $L$ and $M$ are any two line bundles over $W$ then

$$
P(\mathrm{~L})=P(\mathrm{M})=W .
$$

EXAMPLE 5.4. - If $\mathrm{G}=\mathrm{E}_{1} \oplus \mathrm{E}_{2}$, where $\mathrm{E}_{1}=\mathrm{E}_{\mathrm{a}}=\mathrm{E}$, then $\mathrm{B}_{1}=\mathrm{N}\left(P\left(\mathrm{E}_{1}\right), P(\mathrm{G})\right)$ and $\mathrm{B}_{2}=\varrho_{\mathrm{x}}^{*} \mathrm{E}$ have the same projective bundles.

Indeed $P\left(\mathrm{~B}_{2}\right)=P(\mathrm{E}) \times{ }_{W} P(\mathrm{E})$, and the first projection $q_{1}$ is the fibre projection $\varrho_{\mathrm{B}_{2}}$. Then $\check{\mathbf{L}}_{\mathrm{B}_{2}}=q_{2}^{*} \check{\mathrm{L}}_{\mathrm{E}}$. 
On the other hand $Z_{1}=s_{1} P\left(\mathrm{~B}_{2}\right)$ is the result, in fig. 4.1, of blowing up $P(\mathrm{G})$ along $P\left(\mathrm{E}_{1}\right)$ (and also along the disjoint $P\left(\mathrm{E}_{2}\right)$ ). So also $Z_{1}=P\left(\mathrm{~B}_{1}\right)$ where $\mathrm{B}_{1}=\mathrm{N}\left(P\left(\mathrm{E}_{1}\right), P(\mathrm{G})\right)$. In view of this $\check{\mathrm{L}}_{\mathrm{B}_{1}}$ is the normal bundle of $Z_{\mathrm{x}}$ in $H$ (lemma 3.5). So $s_{1}^{*} c_{1}\left(\check{\mathrm{L}}_{\mathrm{B}_{1}}\right)=q_{1}^{*} c_{1}\left(\mathrm{~L}_{\mathrm{E}}\right)-q_{2}^{*} c_{1}\left(\mathrm{~L}_{\mathrm{E}}\right)$, by Theorem 4.1 (iii).

If now, as we did in deriving (5.1) and (5.2), we identify $P\left(B_{1}\right)$ and $P\left(B_{2}\right)$ we must take $s_{1}$ as the identity and $q_{1}=\varrho_{\mathrm{B}}$.

So we get $e_{1}\left(\breve{\mathrm{L}}_{\mathrm{B}_{1}}\right)-e_{1}\left(\breve{\mathrm{L}}_{\mathrm{B}_{2}}\right)=\varrho_{\mathrm{B}}^{*} e_{1}\left(\mathrm{~L}_{\mathrm{E}}\right)$, and in accordance with (5.2) we must have

$$
\mathrm{B}_{1}=\mathrm{B}_{2} \otimes \mathrm{L}_{\mathrm{E}} \quad \text { or } \quad \mathrm{N}\left(P\left(\mathrm{E}_{1}\right), P(\mathrm{G})\right)=\varrho^{*} \mathrm{E} \otimes \mathrm{L}_{\mathrm{E}} .
$$

We shall be able to check this result later.

EXAMPLE 5.5. - Here we introduce the bundle of tangent vectors $\mathbf{E}^{r}$ (or $\mathbf{T}^{\prime}(P(\mathrm{E}))$ ) along the fibres of $P(\mathbf{E})$. We have the exact sequence

$$
0 \rightarrow \mathrm{E}^{\prime} \rightarrow \mathrm{T}(P(\mathrm{E})) \rightarrow \varrho^{*} \mathrm{~T}(W) \rightarrow 0,
$$

where $W$ is the base variety (cf. [3], § 8.1.3).

There is another exact sequence we wish to consider which arises because $\check{\mathrm{L}}_{\mathrm{E}}$ is a sub-bundle of $\varrho^{*} \mathrm{E}$. If we denote the quotient bundle (ef. GrotHendIEcK [7]) by $\mathrm{E}^{(1)}$ we have the sequence

$$
0 \rightarrow \check{\mathrm{L}}_{\mathrm{E}} \rightarrow \varrho^{*} \mathrm{E} \rightarrow \mathrm{E}^{(1)} \rightarrow 0 .
$$

The bundle $P\left(\mathrm{E}^{(1)}\right)$ is the following. If $z$ is a point of $P(\mathrm{E})$ lying over the point $w$ of $W\left(\varrho_{\mathrm{E}} z=w\right)$, then $P\left(\mathrm{E}^{(1)}\right)_{z}$ is isomorphic with the aggregate of planes of $\mathrm{E}_{w}$ (or $\left(Q^{*} \mathrm{E}\right)_{z}$ ) containing the line of $\mathrm{E}_{w}$ which corresponds to the point $z$ of $P(\mathrm{E})$. So $P\left(\mathrm{E}^{(1)}\right)_{z}$ is isomorphic with the lines of $P(\mathrm{E})_{w}$ through the point $z$. Thus $P\left(\mathrm{E}^{(1)}\right)$ is the bundle of projective spaces representing the directions in the fibres of $P(E)$ at the points of $P(\mathrm{E})$, and this is also $P\left(\mathrm{E}^{\prime}\right)$.

It is, however, easily seen that, though $\mathbf{E}^{\prime}$ and $\mathbf{E}^{(1)}$ have the same projective bundle, they are in fact generally distinct. Indeed if we tensor $\mathrm{E}$ with a line bundle $\mathrm{L}$ we do not affect $P(E)$ and hence do not alter $E^{\prime}$. But on the other hand, by (5.1) and (5.5), we tensor $\mathbf{E}^{(1)}$ with $\varrho^{*} \mathbf{L}$. So all we know is that $\mathbf{E}^{\prime}$ is $\mathbf{E}^{(1)}$ tensored with a line bundle.

In fact we shall prove in the next section (the result is known, ef. Popteous [13], p. 292) that

$$
E^{\prime}=E^{(1)} \otimes L_{E}
$$

which, taken with (5.5), gives us the exact sequence

$$
0 \rightarrow \mathbf{1}_{F(\mathrm{E})} \rightarrow \varrho^{*} \mathrm{E} \otimes \mathrm{L}_{\mathrm{E}} \rightarrow \mathrm{E}^{\prime} \rightarrow 0
$$

Alternatively we can calculate $C\left(t, \mathrm{E}^{(1)}\right)$ from (5.5) (the result is given in $\S 7$ ) and we could also calculate $O\left(t, \mathrm{E}^{\prime}\right)$ from (5.4) if we assume the known formula for $C(t, P(\mathrm{E}))$. However, we prefer to deduce $C(t, P(\mathrm{E}))$ from our essentially geometric derivation of (5.6) and (5.7). 
We shall conclude this section with an important result on the bundle $\mathrm{E}^{\prime}$. But first we need a lemma on pull-backs of projective bundles. Suppose $f: U \rightarrow W$ is an arbitrary morphism and $\mathrm{F}=f^{*} \mathrm{E}$ is the pull-back to $U$ of the bundle $\mathrm{E}$ over $W$. Then $P(\mathbf{F})=f^{*} P(\mathbb{E})$ and we have a commutative diagram (5.8), where $\bar{f}$ is the obvious mapping of $P(\mathbf{F})$ on $P(\mathbb{E})$.

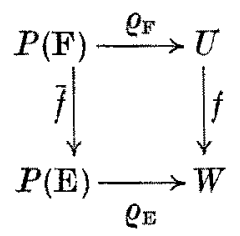

The mapping $\bar{f}$ was introduced by GROTHENDIECK [7, p. 139] in remarking that $\check{\mathrm{L}}_{\mathrm{F}}=\bar{f}^{*} \check{\mathrm{L}}_{\mathrm{E}}$. Here we require the equally natural

LewDa 5.6. - The bundle $\mathrm{F}^{\prime}$ (of tangents along the fibres of $P(\mathrm{~F})$ ) is the pull-back by $\bar{f}$ of $\mathbf{E}^{\prime}$, i.e. $\mathbf{F}^{\prime}=\bar{f}^{*} \mathbf{E}^{\prime}$.

The verification of this natural result is straightforward and we omit it. If we now consider the special case where $U=P(\mathrm{E}), f=\varrho_{E}$, then $P(\mathrm{~F})=P\left(\varrho^{*}(\mathrm{E})\right)=$ $=P(\mathrm{E}) \times_{W} P(\mathrm{E})$ with $\varrho_{F}=q_{1}$ and $\bar{f}=q_{2}$. In this case Lemma 5.6 becomes

Lemina 5.7. - If $\mathrm{F}=\varrho^{*} \mathrm{E}$ and we take $P(\mathrm{~F})=P(\mathrm{E}) \times_{W} P(\mathrm{E})$ with $q_{1}$ as bundle projection $\varrho_{\mathrm{F}}$, then $\mathrm{F}^{\prime}=q_{2}^{*} \mathrm{E}^{\prime}$.

We now use this to show that $\mathrm{E}^{\prime}$ is naturally isomorphic with the normal bundle of the diagonal $\Delta$ on $P(\mathrm{E}) \times_{W} P(\mathrm{E})$. Let $i: \Delta \hookrightarrow P(\mathrm{E}) \times_{W} P(\mathrm{E})$ be the inclusion mapping, and let $\delta$ be the diagonal mapping $\delta: P(\mathrm{E}) \rightarrow \Delta$ and $\varepsilon$ the inverse isomorphism $\varepsilon: \Delta \rightarrow P(\mathrm{E})$. We now have the commutative diagram

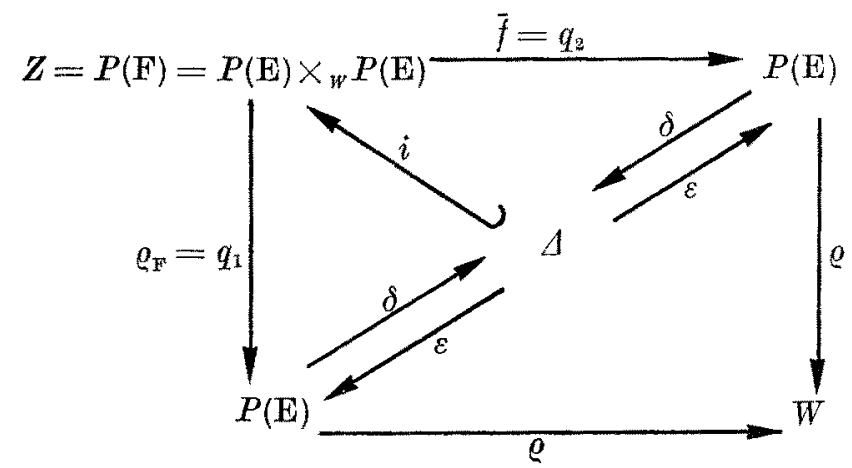

Fig. 5.9.

with $q_{1} i=q_{2} i=\varepsilon, \varepsilon=\delta^{-1}$. This gives us

$$
i^{*} q_{1}^{*}=i^{*} q_{2}^{*}=\varepsilon^{*}, \quad \varepsilon^{*} \delta^{*}=\delta^{*} \varepsilon^{*}=\mathrm{Id} .
$$

We can now establish

LEMMA 5.8. $-i * \mathrm{~T}(Z)=\mathrm{T}(\Delta) \oplus \varepsilon^{*} \mathrm{E}^{\prime}$. 
This implies that the normal bundle sequence of $\Delta$ in $P(\mathrm{E}) \times_{W} P(\mathrm{E})$ splits and that the normal bundle is naturally isomorphic with $\varepsilon^{*} \mathbf{E}^{\prime}$.

Proor. - Consider the bundle along the fibres sequence

$$
0 \rightarrow \mathrm{F}^{\prime} \rightarrow \mathrm{T} P(\mathrm{~F}) \rightarrow \varrho_{\mathrm{F}}^{*} \mathrm{~T}(P(\mathrm{E})) \rightarrow 0 .
$$

Now replace (lemma 5.7) $\mathrm{F}^{\prime}$ with $q_{2}^{*} \mathrm{E}^{\prime}$, $\varrho_{\mathrm{F}}^{*}$ by $q_{1}^{*}$, operate on the sequence with $i^{*}$ and use (5.10). We now get

$$
0 \rightarrow \varepsilon^{*} \mathbf{E}^{\prime} \rightarrow i * \mathrm{~T}(Z) \rightarrow \varepsilon^{*} \mathrm{~T}(P(\mathrm{E})) \rightarrow 0 .
$$

But $\varepsilon^{*}$ is an isomorphism, so $\varepsilon^{*} \mathrm{~T}(P(\mathrm{E}))=\mathrm{T}(A)$ which is a sub-bundle of $i^{*} \mathrm{~T}(Z)$. So the sequence (5.11) splits and we have

$$
i^{*} \mathbf{T}(Z)=\mathbf{T}(\Delta) \oplus \varepsilon^{*} \mathbf{E}^{\prime} .
$$

But we also have the normal bundle sequence

$$
0 \rightarrow \mathbf{T}(\Delta) \rightarrow i^{*} \mathbf{T}(Z) \rightarrow \mathrm{N}(\Delta, Z) \rightarrow 0,
$$

so that $\mathrm{N}(\Delta, Z)=\varepsilon^{*} \mathrm{E}^{\prime}$. Operating on this with $\delta^{*}$ we get

THEOREM 5.9. $-\mathbf{E}^{\prime}=\delta^{*} \mathrm{~N}(\Delta, Z)$.

\section{6. - Projective closures of vector bundles.}

If $\mathrm{G}=\mathrm{E} \oplus 1_{w}$ we have defined the projective closure $\vec{E}$ of $\mathrm{E}$ to be the bundle $P(\mathrm{G})$. Note that this is the same as the bundle $P\left(\mathrm{G}^{\dagger}\right)$ where $\mathrm{G}^{\dagger}=(\mathrm{E} \otimes \mathrm{L}) \oplus \mathrm{L}$ for any line-bundle L. Alternatively the bundle $P(\mathrm{E} \oplus \mathrm{L})$ is the projective closure of $\mathrm{E} \otimes \check{\mathrm{L}}$.

The bundle $P(\mathrm{G})$ includes sub-bundles $P(\mathrm{E})$ and $W=P(1)$ as described in $\S 4$. We also consider the bundle $H=P(\mathrm{~S})=P\left(\breve{\mathrm{L}}_{\mathrm{x}} \oplus 1\right)$ over $P(\mathrm{E}) \times_{W} P(1)=P(\mathrm{E})$ which is obtained (as in $\S 4$ ) from $P(G)$ in this case by blowing up along $W$.

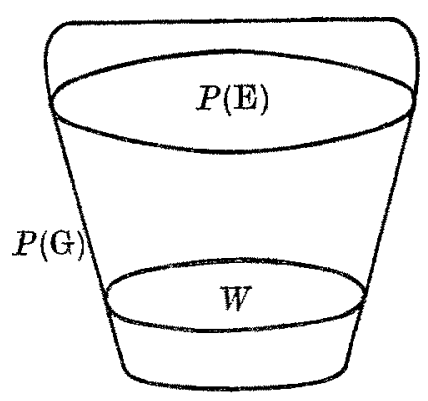

Fig. 6.2.

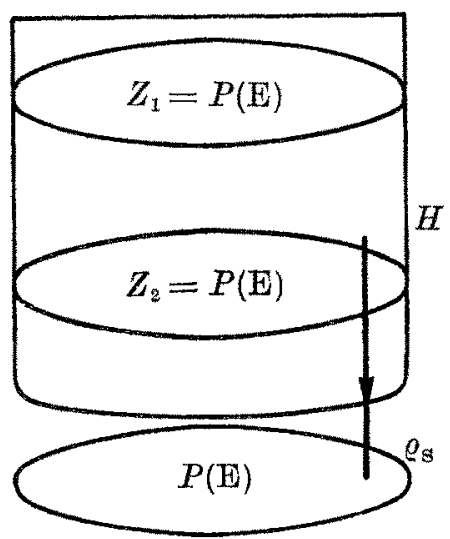


We list here some useful formulae: the first of them is Theorem 4.2 and the other two are Theorem 4.1 , (i) and (iii). We state them as

$$
\text { LEMMA 6.1. }-\mathrm{cl}_{P(\mathbb{Q})} P(\mathrm{E})=\xi_{\mathrm{q}}, \mathrm{cl}_{\sharp} Z_{1}=\xi_{\mathrm{s}}, \mathrm{cl}_{\sharp} Z_{2}=\xi_{\mathrm{s}}-\varrho_{\mathrm{s}}^{*} \xi_{\mathrm{B}} \text {. }
$$

The "special case " of blowing up a subvariety which we shall treat in $\S 8$, and from which the solution of the general problem will be derived, is obtained by taking $X=P(\mathrm{G}), Y=W, H=X^{\prime}$ and $Z_{2}=Y^{\prime}$, i.e. we blow up $P(\mathrm{G})$ along $W=P(1)$.

The variety $P(\mathrm{G}) \backslash P(\mathrm{E})$ is isomorphic with $\mathrm{E}$ (if it were not there would be little point in defining $\bar{E}$ to be $P(\mathrm{G}))$ : to demonstrate this isomorphism we need only globalise the isomorphism described (in the fibres) at the end of $\$ 2$.

Looking at $P(G)$ "the other way up " we can consider $P(G) \backslash W$ which is a linebundle over $P(E)$. But this is isomorphic with $H \backslash Z_{2}$ which is the same line-bundle $L$ over $Z_{1}$.

But (lemma 3.3(e)) $\mathrm{L}=\mathrm{N}(P(\mathbf{E}), L)=\mathrm{N}\left(Z_{1}, H\right)$. Hence, by Theorem 4.1 (iii), $e_{1}(\mathrm{~L})=\xi_{\mathrm{E}}$, so that $\mathrm{L}=\mathrm{L}_{\mathrm{E}}$. We summarise all this in the useful

THEOREM 6.2. - If $\bar{E}=P(\mathrm{G})=P(\mathrm{E} \oplus 1)$, then $\bar{E} \backslash P(\mathrm{E})$ is isomorphic with the bundle $\mathrm{E}$ and $\bar{E} \backslash W$ is isomorphic with the line bundle $\mathrm{L}_{\mathrm{E}}$.

Note that $H=P\left(\check{\mathrm{L}}_{\mathrm{E}} \oplus 1_{P(\mathrm{E})}\right)=P\left(1_{P(\mathrm{E})} \oplus \mathrm{L}_{\mathrm{E}}\right)$. Thus

LEMma 6.3. $-H \backslash Z_{1}$ is the bundle $\check{\mathrm{L}}_{\mathrm{E}}$ over $Z_{2}, H \backslash Z_{2}$ is the bundle $\mathrm{L}_{\mathrm{E}}$ over $Z_{1}$.

(It is clear why "turning the line-bundle upside down " dualises it: for if we invert all local coordinates we also invert the coordinate transformations.)

This last idea supplies a rapid verification of the last two parts of Theorem 4.1. In the context of that theorem $\mathrm{G}$ is now $\mathrm{E}_{1} \oplus \mathrm{E}_{2}, H=P(\mathrm{~S})=P\left(q_{1}^{*} \check{\mathrm{L}}_{\mathrm{E}_{1}} \oplus q_{2}^{*} \check{\mathrm{L}}_{\mathrm{E}_{2}}\right)$. So we have

$$
H=P\left(1 \oplus\left(q_{\mathrm{I}}^{*} \mathrm{~L}_{\mathrm{E}_{1}} \otimes q_{2}^{*} \check{\mathrm{L}}_{\mathrm{E}_{2}}\right)\right)=P\left(\left(q_{1}^{*} \breve{\mathrm{L}}_{\mathrm{E}_{1}} \otimes q_{2}^{*} \mathrm{~L}_{\mathrm{E}_{2}}\right) \oplus 1\right)
$$

The first form for $H$ shows that $H \backslash Z_{2}$ is isomorphic with the bundle $q_{1}^{*} \mathrm{~L}_{\mathrm{E}_{1}} \otimes q_{2}^{*} \check{\mathrm{L}}_{\mathrm{E}_{2}}$ over $Z_{1}$ and the second that $H \backslash Z_{1}$ is isomorphic with the bundle $q_{1}^{*} \check{L}_{\mathrm{E}_{1}} \otimes q_{2}^{*} \mathrm{~L}_{\mathrm{E}_{\mathrm{a}}}$ over $Z_{2}$. (Again turning upside down dualises.) Thus we have shown that (again applying lemma $3.3(c))$.

$$
\mathrm{N}\left(Z_{1}, H\right)=q_{1}^{*} \mathrm{~L}_{\mathrm{E}_{1}} \otimes q_{2}^{*} \check{\mathrm{L}}_{\mathrm{E}_{2}}, \quad \mathrm{~N}\left(Z_{2}, H\right)=q_{1}^{*} \check{\mathrm{L}}_{\mathrm{E}_{1}} \otimes q_{2}^{*} \mathrm{~L}_{\mathrm{E}_{2}},
$$

giving $t_{1}\left(Z_{1}, H\right)=\xi_{1}-\xi_{2}, t_{1}\left(Z_{2}, H\right)=\xi_{2}-\xi_{1}$ as before

Let us now consider the bundle $P\left(\varrho^{*} \mathrm{E} \oplus \check{\mathrm{L}}_{\mathrm{E}}\right)$, a bundle over $P(\mathrm{E})$ which we shall denote for brevity by $V$. This is, of course, the bundle $\overline{Q^{*} \mathrm{E} \otimes \mathrm{L}_{\mathrm{E}}}$. The variety $V$ contains the sub-bundles $U=P\left(\varrho^{*} \mathrm{E}\right)=P(\mathrm{E}) \times_{W} P(\mathrm{E})$ and $P\left(\check{\mathrm{L}}_{\mathrm{E}}\right)=P(\mathrm{E})$ : $V$ is the join of these sub-bundles. We have already remarked in Example 5.3 that $\varrho_{e^{*} \mathrm{E}}=q_{1}$, and $\mathrm{L}_{e^{* \mathrm{E}}}=q_{2}^{*} \mathrm{~L}_{\mathrm{E}}$. 
But $U$ also contains the diagonal sub-bundle $\Delta$ corresponding to the inclusion of $\check{\mathrm{L}}_{\mathrm{E}}$ in $\varrho_{\mathrm{E}}^{*} \mathrm{E}$. There is also the bundle $K=P\left(\breve{\mathrm{L}}_{\mathrm{E}} \oplus \breve{\mathrm{L}}_{\mathrm{E}}\right)$ which contains $\Delta$ and $P(\mathrm{E})$ as subvarieties. Note that $K=P(\mathbf{1} \oplus \mathbf{1})=P(\mathrm{E}) \times \mathbb{P}^{1}$ and is also $\overline{\mathbf{1}}_{P(\mathbb{E})}$.

We now have the following figure.

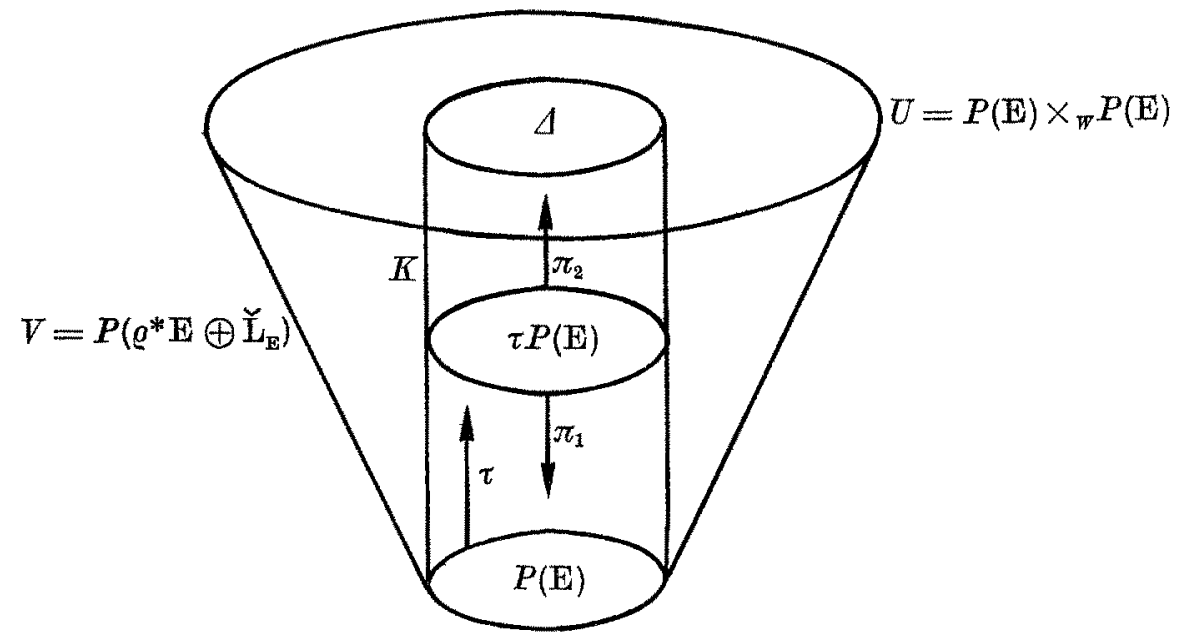

Fig. 6.2.

Let $j_{1}: P(\mathrm{E}) \hookrightarrow K$ and $j_{2}: \Delta \hookrightarrow K$ be the inclusions. As before we have the isomorphism $\delta: P(\mathrm{E}) \stackrel{\sim}{\rightarrow} \Delta$ and the inclusion $i: \Delta \hookrightarrow U$ (ef. (5.9)).

We shall consider a section of $K$, say $\tau: P(\mathrm{E}) \rightarrow K$, different from both $j_{1}$ (i.e. $P(\mathbf{E}))$ and $j_{2} \delta$ (i.e. $\Delta$ ).

Let $K_{1}=K \backslash \Delta, K_{2}=K \backslash P(\mathrm{E})$. Then $\tau P(\mathrm{E}) \subset K_{1} \cap K_{2}$.

Let $V_{1}=\bigvee U, V_{2}=V \backslash P(\mathrm{E})$. Then $K_{1}, K_{2}, V_{1}, V_{2}$ are vector bundles where

$$
K_{1}=1_{P(E)}, \quad K_{2}=1_{A},
$$

by Lemma 6.3. Also by Theorem 6.2

$$
V_{1}=\varrho^{*} \mathrm{E} \otimes \mathrm{L}_{\mathrm{E}}, \quad V_{2}=\mathrm{L}_{e^{*} \mathrm{E} \otimes \mathrm{L}_{\mathrm{E}}} .
$$

But, by dualising (5.1), $\mathrm{L}_{e^{*} \otimes \mathrm{L}_{\mathrm{E}}}=\mathrm{L}_{e^{*} \mathrm{E}} \otimes q_{1}^{*} \check{\mathrm{L}}_{\mathrm{E}}=q_{2}^{*} \mathrm{~L}_{\mathrm{E}} \otimes q_{1}^{*} \check{\mathrm{L}}_{\mathrm{E}}$.

Now, as $\check{\mathrm{L}}_{\mathrm{E}} \subset \varrho^{*} \mathrm{E}, K_{1}$ is the sub-bundle of $V_{1}$ corresponding to $\check{\mathrm{L}}_{\mathrm{E}} \otimes \mathrm{L}_{\mathrm{E}}$. Hence, by lemma $3.3(b)$, writing $\pi_{1}$ (and $\pi_{2}$ ) for $\pi_{\mathrm{K}_{1}}$ (and $\pi_{\mathrm{K}_{2}}$ ),

$$
\mathrm{N}\left(K_{1}, V_{1}\right)=\pi_{1}^{*}\left(\varrho^{*} \mathrm{E} \otimes \mathrm{L}_{\mathrm{E}} / 1_{P(\mathrm{E})}\right)=\pi_{1}^{*}\left(\mathrm{E}^{(1)} \otimes \mathrm{L}_{\mathrm{E}}\right),
$$

because $\varrho^{*} \mathrm{E} \otimes \mathrm{L}_{\mathrm{E}} / 1_{\left.P^{\prime} \mathrm{E}\right)}=\left(\varrho^{*} \mathrm{E} / \check{\mathrm{L}}_{\mathrm{E}}\right) \otimes \mathrm{L}_{\mathrm{E}}$.

Also $\Delta$ is the part of $U$ for which $q_{1}=q_{2}$. Thus $V_{2}$ induces the trivial linebundle, i.e. $K_{2}$ on $\Delta$. So by lemma 3.3(a)

$$
\mathrm{N}\left(K_{2}, V_{2}\right)=\pi_{2}^{*} \mathrm{~N}(\Delta, U) \text {. }
$$


But $\pi_{1} \tau=\mathrm{Id}_{P(\mathbb{B})}, \pi_{2} \tau=\delta$, so

$$
\tau^{*} \mathrm{~N}(K, V)=\tau^{*} \mathrm{~N}\left(K_{1}, V_{1}\right)=\tau^{*} \pi_{1}^{*}\left(\mathrm{E}^{(1)} \otimes \mathrm{L}_{\mathrm{E}}\right)=\mathrm{E}^{(1)} \otimes \mathrm{L}_{\mathrm{E}},
$$

and

$$
\tau^{*} \mathrm{~N}(K, V)=\tau^{*} \mathrm{~N}\left(K_{2}, V_{2}\right)=\tau^{*} \pi_{2}^{*} \mathrm{~N}(\Delta, U)=\delta^{*} \mathrm{~N}(\Delta, U)=\mathrm{E}^{\prime},
$$

by Theorem 5.9.

By equating these two values of $\tau * \mathrm{~N}(K, V)$ we have thas established (5.6), and hence (5.7), which we record as

THEOREM 6.4. $-\mathrm{E}^{\prime}=\mathrm{E}^{(1)} \otimes \mathrm{L}_{\mathrm{E}}$

and

THEOREM 6.5. - There is an exact sequence

$$
0 \rightarrow \mathbf{1}_{P(\mathrm{E})} \rightarrow \varrho^{*} \mathrm{E} \otimes \mathrm{L}_{\mathrm{E}} \rightarrow \mathrm{E}^{\prime} \rightarrow 0,
$$

where the map $1_{P(E)} \rightarrow \varrho^{*} \mathrm{E} \otimes \mathrm{L}_{\mathrm{E}}$ derives from the inclusion $\breve{\mathrm{L}}_{\mathrm{E}} \subset \varrho^{*} \mathrm{E}$ by tensoring with $\mathrm{L}_{\mathrm{E}}$.

\section{7. - Some formulae for tangent and normal bundles.}

In this section we give some applications of theorems 6.4 and 6.5. Some of the results are not new, but the derivation we give of them here is of some intrinsic interest.

Theoram 7.1. $-\bar{C}\left(t, \mathrm{E}^{\prime}\right)=\bar{C}\left(t+\xi_{\mathrm{E}}, \mathrm{E}^{(1)}\right)=t^{-1}\left[\varphi^{*} \bar{C}\right]\left(t+\xi_{\mathrm{E}}, \mathrm{E}\right)$.

(Note that $\left[\varrho^{*} \bar{C}\right]\left(t+\xi_{\mathrm{E}}, \mathrm{E}\right)$ is actually divisible by $t$ in accordance with (3.10).)

Starting from the exact sequence (5.5)

$$
0 \rightarrow \check{\mathrm{L}}_{\mathrm{E}} \rightarrow \varrho^{*} \mathrm{E} \rightarrow \mathrm{E}^{(1)} \rightarrow 0
$$

we get, by (3.5),

$$
\left[\varrho^{*} C\right](t, \mathrm{E})=O\left(t, \varrho^{*} \mathrm{E}\right)=O\left(t, \check{\mathrm{L}}_{\mathrm{E}}\right) \cdot C\left(t, \mathrm{E}^{(1)}\right),
$$

or

$$
\left[\varrho^{*} C\right](t, \mathrm{E})=\left(1-\xi_{\mathrm{E}} t\right) C\left(t, \mathrm{E}^{(1)}\right)
$$

So

$$
\left[\varrho^{*} \bar{C}\right](t, \mathrm{E})=\left(t-\xi_{E}\right) \bar{C}\left(t, \mathrm{E}^{(1)}\right),
$$

i.e.

$$
\left[\varrho^{*} \bar{C}\right]\left(t+\xi_{E}, \mathbf{E}\right)=t \bar{C}\left(t+\xi_{E}, \mathbf{E}^{(1)}\right) .
$$

Thus $\vec{C}\left(t+\xi_{\mathrm{E}}, \mathrm{E}^{(1)}\right)=t^{-1}\left[e^{*} \bar{C}\right]\left(t+\xi_{\mathrm{E}}, \mathrm{E}\right)$. 
But, by theorem 6.4 and lemma (5.2)

$$
\bar{C}\left(t, \mathbf{E}^{\prime}\right)=\bar{C}\left(t, \mathbf{E}^{(1)} \otimes \mathrm{L}_{\mathrm{E}}\right)=\bar{C}\left(t+\xi_{\mathrm{E}}, \mathrm{E}^{(1)}\right)
$$

and the theorem is established.

THEOREM 7.2. $\left.-\bar{C}(t, P(\mathrm{E}))=t^{-1}\left[\varrho^{*} \bar{C}\right]\left(t+\xi_{\mathrm{E}}, \mathrm{E}\right) \cdot\left[Q^{*} \bar{C}\right](t, W)\right)$.

This is an immediate consequence of the exact sequence (5.4).

The most important result of this section is

THEOREM 7.3. - Suppose $V$ is a subvariety of $W$ with inclusion mapping $i: \nabla \hookrightarrow W$. Let $\mathrm{E}$ be a vector bundle over $W$ and $\mathrm{F}$ a sub-bundle of $i^{*} \mathrm{E}$. Then there is an exact sequence (of bundles over $P(\mathrm{~F})$ )

$$
0 \rightarrow \varrho_{F}^{*}\left(i^{*} \mathrm{E} / \mathrm{F}\right) \otimes \mathrm{L}_{\mathrm{F}} \rightarrow \mathrm{N}(P(\mathrm{~F}), P(\mathrm{E})) \rightarrow \varrho_{F}^{*} \mathrm{~N}(V, W) \rightarrow 0
$$

There are two special cases which we state as corollaries. If we take $W=V$ we get the following

Corollary 1. - If $\mathbf{F}$ is a sub-bundle of the bundle $\mathbf{E}$ over $W$ then

$$
\varrho_{\mathrm{F}}^{*}(\mathrm{E} / \mathrm{F}) \otimes \mathrm{L}_{\mathrm{F}}=\mathrm{N}(P(\mathrm{~F}), P(\mathrm{E})) \text {. }
$$

(This is a generalisation of formula 5.3, the two bundles in that case being $\mathbf{E} \oplus \mathrm{E}$ and its first component.)

The other special case comes by taking $\mathrm{F}=i^{*} \mathrm{E}$, giving

COROLlARX 2. - If $V$ is a subvariety of $W$ with inclusion $i: V \hookrightarrow W$, then a bundle $\mathrm{E}$ over $W$ gives rise to an isomorphism

$$
N\left(P\left(i^{*} \mathrm{E}\right), P(\mathrm{E})\right) \simeq \varrho_{i^{*} \mathrm{E}}^{*} \mathrm{~N}(\nabla, W) .
$$

It is no more trouble to establish the theorem directly than to build it up from the two special cases, so we shall now proceed with the proof of Theorem 7.3. Let $j$ be the inclusion $j: P(\mathrm{~F}) \hookrightarrow P(\mathrm{E})$ giving the commutative diagram

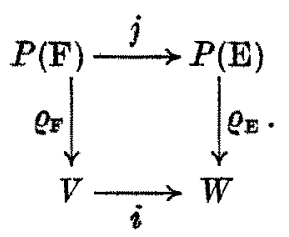

From the exact sequence (5.4)

$$
0 \rightarrow \mathrm{E}^{\prime} \rightarrow \mathrm{T}(P(\mathrm{E})) \rightarrow \varrho_{\mathrm{E}}^{*} \mathrm{~T}(W) \rightarrow 0,
$$


and because (7.1) $j^{*} \varrho_{\mathrm{E}}^{*}=\varrho_{\mathrm{F}}^{*} i^{*}$ we get, by operating with $j^{*}$, the middle row of the following commutative diagram; the first row is a direct application of (5.4)

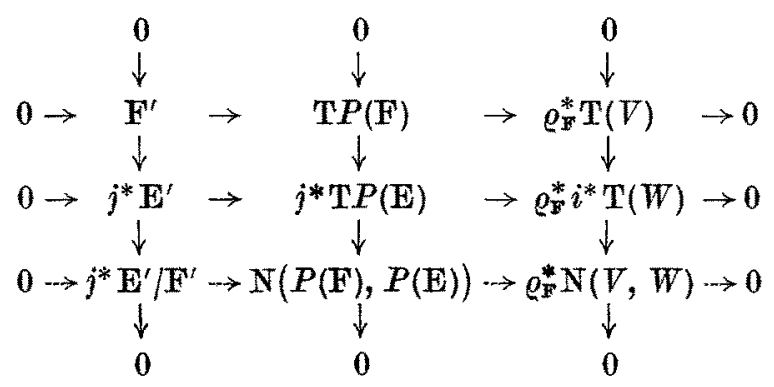

and the exactness of the dotted row is a consequence of the exactness of the columns and the other rows.

We now get an alternative expression for the first term in the bottom row of (7.2). We use the commutative diagram below in which the first two rows are derived from Theorem 6.5 (and the second one is operated on by $j^{*}$, again using the result $j^{*} \varrho_{\mathrm{E}}^{*}=\varrho_{\mathrm{F}}^{*} i^{*}$ derived from (7.1)).

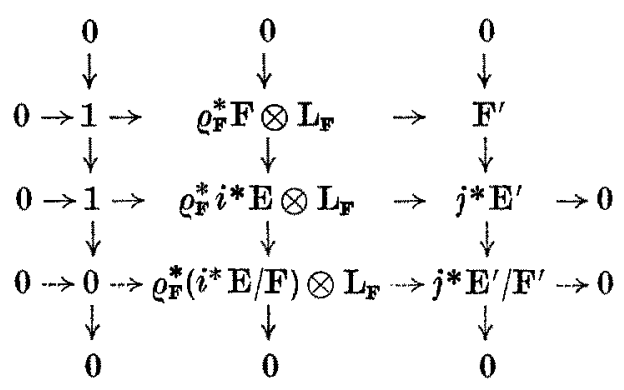

The theorem now follows at once.

\section{8. - Blowing up Chern classes. The special case.}

We consider now the following situation. Let $Y$ be a variety and $\mathrm{E}$ a vectorbundle over $Y$. Let $\mathrm{G}=\mathrm{E} \oplus \mathbf{1}_{Y}, X=\bar{E}=P(\mathrm{G})$, the projective closure of $E$ and $i: Y \hookrightarrow P(G)$ the inclusion mapping. We have the situation of fig. 8.2 (cf. fig. 6.1).

In this figure $Z_{1}$ and $Z_{2}$ are copies of $P(\mathrm{E}), Z_{1}=\Psi^{-1}(P(\mathrm{E})), Z_{2}=\Psi^{-1}(Y)$, and we shall identify $Z_{2}$ with the base space $P(E)$ of the bundle $H$ and also indentify $Y$ with the base space of the bundle $P(G)$. If we denote by $j$ (instead of $i_{2}$ as previously) the inclusion $j: Z_{2} \hookrightarrow H$, then, because of the identifications just made

$$
\varrho_{\mathrm{s}} j=\operatorname{Id}(\text { on } P(\mathrm{E})), \quad \varrho_{\mathrm{a}} i=\operatorname{Id}(\text { on } Y),
$$

where Id represents the appropriate identical transformation. 


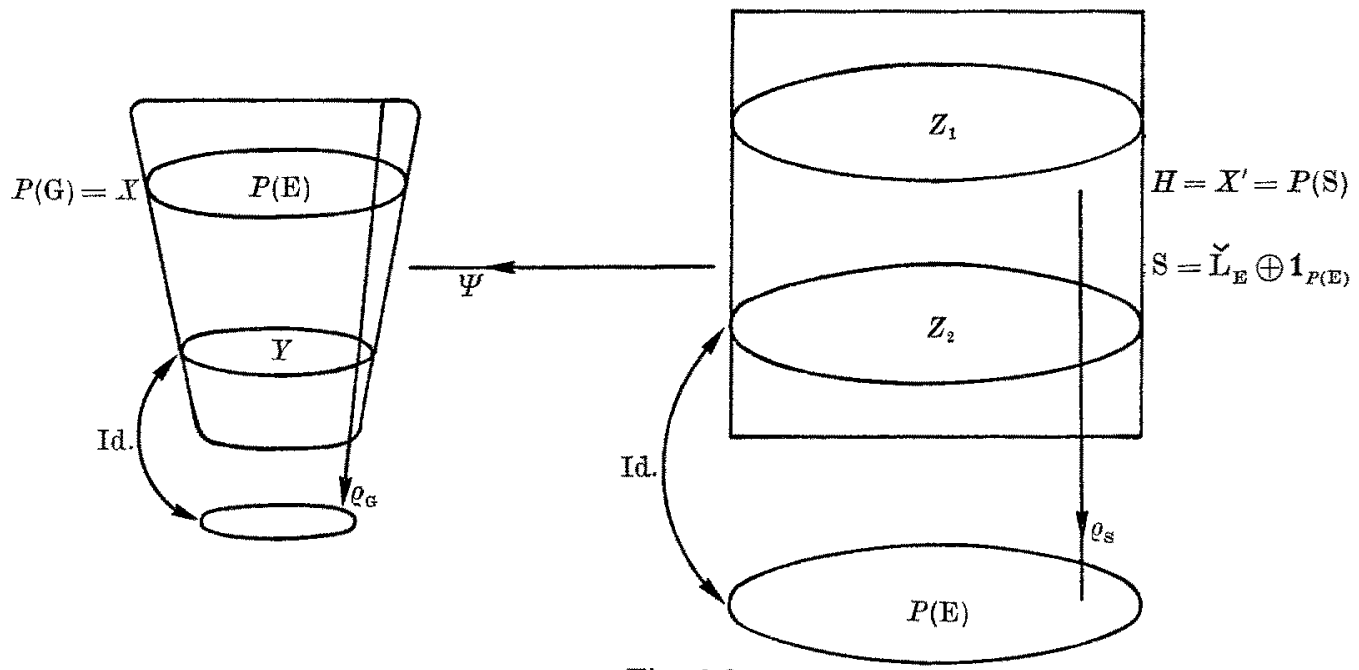

Fig. 8.2.

We have the following blowing-up diagram augmented by the inclusion in it of $\varrho_{\mathrm{s}}$ and $\varrho_{g}$ and of the diagonal morphism $\lambda$ to be introduced in (8.4).

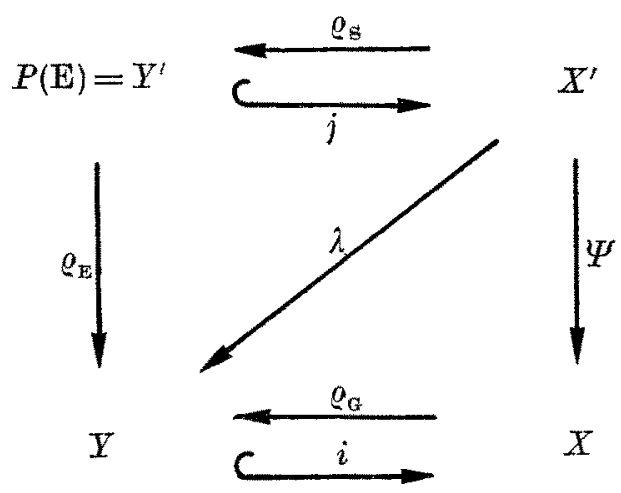

We shall establish the following "Theorem of blowing up Chern classes " in this special case.

THEOREM 8.1.

$\bar{C}\left(t, X^{\prime}\right)-\left[\Psi^{*} \bar{C}\right](t, X)=j_{*}\left\{\frac{-1}{\xi_{\mathrm{E}}}\left[\varrho_{\mathrm{E}}^{*} \bar{C}\right](t, Y)\left\{\left(1-\xi_{\mathrm{E}} t\right)\left[\varrho_{\mathrm{E}}^{*} \bar{C}\right]\left(t+\xi_{\mathrm{E}}, \mathrm{E}\right)-\left[\varrho_{\mathrm{E}}^{*} \bar{C}\right](t, \mathrm{E})\right\}\right\}$.

From diagram 8.3

$$
\varrho_{\mathrm{s}}^{*} \varrho_{\mathrm{E}}^{*}=\Psi^{*} \varrho_{\mathrm{G}}^{*}=\lambda^{*}(\mathrm{say}) \text { and } j^{*} \lambda^{*}=\varrho_{\mathrm{E}}^{*} .
$$

Hence, applying theorem 7.2, we get

$$
\bar{C}\left(t, X^{\prime}\right)=\bar{C}(t, P(\mathbf{S}))=t^{-1}\left[\varrho_{\mathrm{S}}^{*} \bar{C}\right]\left(t+\xi_{\mathrm{s}}, \mathbf{S}\right) \cdot\left[\varrho_{\mathrm{s}}^{*} \bar{C}\right](t, P(\mathbf{E}))
$$


But

$$
\bar{C}(t, S)=t\left(t-\xi_{\mathrm{E}}\right), \quad \bar{C}(t, P(\mathrm{E}))=t^{-1}\left[\varrho_{\mathrm{E}}^{*} \bar{C}\right]\left(t+\xi_{\mathrm{E}}, \mathrm{E}\right) \cdot\left[\varrho_{\mathrm{E}}^{*} \bar{C}\right](t, Y)
$$

Hence

$$
\bar{C}\left(t, X^{\prime}\right)=t^{-1}\left(t+\xi_{\mathrm{s}}\right)\left(t+\xi_{\mathrm{s}}-\varrho_{\mathrm{s}}^{*} \xi_{\mathrm{E}}\right) \cdot t^{-1}\left[\lambda^{*} \bar{C}\right]\left(t+\varrho_{\mathrm{s}}^{*} \xi_{\mathrm{s}}, \mathrm{E}\right) \cdot\left[\lambda^{*} \bar{C}\right](t, Y)
$$

And

$$
\bar{C}(t, X)=\bar{C}(t, P(\mathrm{G}))=t^{-1}\left[\varrho_{\mathrm{G}}^{*} \bar{C}\right]\left(t+\xi_{\mathrm{G}}, \mathrm{G}\right) \cdot\left[\varrho_{\mathrm{Q}}^{*} \bar{C}\right](t, Y)
$$

But, as

$$
\mathbf{G}=\mathbf{E} \oplus \mathbf{1}, \quad \bar{C}(t, \mathrm{G})=t \bar{C}(t, \mathbf{E})
$$

So

$$
\Psi^{*} \bar{C}(t, X)=t^{-1}\left(t+\Psi^{*} \xi_{\mathrm{G}}\right) \cdot\left[\lambda^{*} \bar{C}\right]\left(t+\Psi^{*} \xi_{\mathrm{G}}, E\right) \cdot\left[\lambda^{*} \bar{C}\right](t, \bar{Y})
$$

But, by lemma 6.1, $\xi_{\mathrm{Q}}=\mathrm{el}_{P(\mathrm{G})} P(\mathrm{E})$, so

$$
\Psi *\left(\xi_{\mathrm{a}}\right)=\mathrm{cl}_{\mathrm{H}} Z_{1}=\xi_{\mathrm{s}} \quad \text { and } \quad \xi_{\mathrm{s}}-\varrho_{\mathrm{s}}^{*} \xi_{\mathrm{E}}=\mathrm{cl}_{H} Z_{2}=j_{*}(1)
$$

Thus

$$
\begin{aligned}
& \bar{C}\left(t, X^{\prime}\right)-\left[\Psi^{*} \bar{C}\right](t, X)= \\
= & t^{-2}\left(t+\xi_{\mathrm{s}}\right)\left[\lambda^{*} \bar{C}\right](t, X)\left\{\left(t+j_{*}(1)\right)\left[\lambda^{*} \bar{C}\right]\left(t+\xi_{\mathrm{s}}-j_{*}(1), \mathrm{E}\right)-t\left[\lambda^{*} \bar{C}\right]\left(t+\xi_{\mathrm{s}}, \mathbf{E}\right)\right\} .
\end{aligned}
$$

But $j_{*}(1)$ is a factor of the terms in curly brackets on the right hand side, so every coefficient of $t$ on the R.H.S. is of the form

$$
\xi_{\mathrm{s}}^{k} \cdot \lambda^{*} a \cdot\left(j_{*}(1)\right)^{r}
$$

where $a \in \mathcal{A}(Y), r \geqslant 1$.

Bat we can write (8.7) as

$$
\left.j_{*}(1) \cdot \xi_{*}^{k_{*}} \cdot \lambda^{*} a \cdot\left(j_{*}(1)\right)\right)^{r-1}
$$

and by the projection formula (1.1) this is equal to

$$
j_{*}\left(j^{*} \xi_{s}^{\xi_{*}} \cdot j^{*} \lambda^{*} a \cdot\left(j^{*} j_{*}(1)\right)^{r-1}\right)
$$

But $j^{*} \lambda^{*}=\varrho_{\mathrm{E}}^{*}(8.4), j^{*} j_{*}(1)=j^{*}\left(\xi_{\mathrm{s}}-\varrho_{\mathrm{s}}^{*} \xi_{\mathrm{E}}\right)$.

But $j^{*} \xi_{\mathrm{s}}=0$, since $\xi_{\mathrm{s}}=\mathrm{cl}_{H} Z_{1}$ and so $j^{*} \xi_{\mathrm{s}}$ is the intersection class of $Z_{1}$ and $Z_{2}$ (which have empty intersections).

Thus $j^{*} \xi_{\mathrm{S}}=0$ and $j^{*} j_{*}(1)=-j^{*} \varrho_{\mathrm{S}}^{*} \xi_{\mathrm{E}}=-\xi_{\mathrm{E}}$, by (8.1).

Hence by (8.8)

$$
\xi_{\mathrm{s}}^{k} \cdot \lambda^{*} a \cdot\left(j_{*}(1)\right)^{r}=j_{*}\left(-\frac{1}{\xi_{\mathrm{E}}} \cdot\left(j^{*} \xi_{\mathrm{s}}\right)^{k} \cdot \varrho_{\mathrm{E}}^{*} a \cdot\left(-\xi_{\mathrm{E}}\right)^{r}\right),
$$


which is zero unless $k=0$ and is then

$$
j_{*}\left(-\frac{1}{\xi_{\mathrm{E}}} \cdot \varrho_{\mathrm{E}}^{*} a:\left(-\xi_{\mathrm{E}}\right)^{r}\right)
$$

This means that, in (8.6), we essentially have to precede the R.H.S. with $j_{*}$, replace $\lambda^{*}$ by $\varrho_{\mathrm{E}}^{*}$, replace $\xi_{\mathrm{s}}$ by zero, replace $-j_{*}(1)$ by $\xi_{\mathrm{E}}$ and multiply the result by $-1 / \xi_{\mathrm{E}}$. Doing this we get

$$
\begin{aligned}
\bar{C}\left(t, X^{\prime}\right)-[\Psi * \bar{C}](t, X) & =j_{*}\left\{-\frac{1}{t \xi_{\mathrm{E}}}\left[\varrho_{\mathrm{E}}^{*} \bar{C}\right](t, Y)\left\{\left(t-\xi_{\mathrm{E}}\right)\left[\varrho_{\mathrm{E}}^{*} \bar{C}\right]\left(t+\xi_{\mathrm{E}}, \mathrm{E}\right)-t\left[\varrho_{\mathrm{E}}^{*} \bar{C}\right](t, \mathrm{E})\right\}\right\} \\
& =j_{*}\left\{-\frac{1}{\xi_{\mathrm{E}}}\left[\varrho_{\mathrm{E}}^{*} \bar{C}\right](t, Y)\left\{\left(1-\xi_{\mathrm{E}} / t\right)\left[\varrho_{\mathrm{E}}^{*} \bar{C}\right]\left(t+\xi_{\mathrm{E}}, \mathrm{E}\right)-\left[\varrho_{\mathrm{E}}^{*} \bar{C}\right](t, \mathrm{E})\right\}\right\}
\end{aligned}
$$

and the theorem is established.

It is perhaps worth stating specifically as a corollary the more usual form of the result, in terms of total Chern classes, obtained from the above by putting $t=1$.

THEOREM 8.2.

$$
\boldsymbol{c}\left(X^{\prime}\right)-\Psi^{*} \boldsymbol{c}(X)=j_{*}\left\{\frac{-1}{\xi_{\mathrm{E}}} e_{\mathrm{E}}^{*} \boldsymbol{c}(Y)\left\{\left(1-\xi_{\mathrm{E}}\right)\left[\varrho_{\mathrm{E}}^{*} \vec{C}\right]\left(1+\xi_{\mathrm{E}}, \mathrm{E}\right)-\varrho_{\mathrm{E}}^{*} \boldsymbol{c}(\mathrm{E})\right\}\right\} .
$$

\section{9. - Blowing up Chern classes. The general case.}

Let us now consider a variety $X$ and a subvariety $Y$ (of co-dimension at least 2 ), both assumed non-singular, which we shall blow up to get a birational transform $X^{\prime}$ of $X$ with a primal $Y^{\prime}$ on $X^{\prime}$ which arises from "blowing up " $Y$. Let us denote by $\mathbf{E}$ the normal bundle $\mathrm{N}(Y, X)$. We have the blowing up diagram (cf. 3.2)

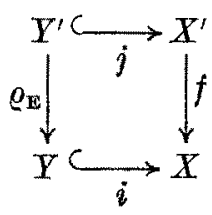

where $Y^{\prime}=P(\mathrm{E}), \mathrm{N}\left(Y^{\prime}, X^{\prime}\right)=\breve{\mathrm{L}}_{\mathrm{E}}$ and $f$ is a birational morphism.

We propose to establish the following blowing up Theorems, exactly the same results as obtained in $\S 8$ for the special case.

THEOREM 9.1.

$\bar{C}\left(t, X^{\prime}\right)-\left[f^{*} \bar{C}\right](t, X)=j_{*}\left\{-\frac{1}{\xi_{\mathrm{E}}}\left[\varrho_{\mathrm{E}}^{*} \bar{C}\right](t, Y)\left\{\left(1-\xi_{\mathrm{E}}(t)\left[\varrho_{\mathrm{E}}^{*} \bar{C}\right]\left(t+\xi_{\mathrm{E}}, \mathrm{E}\right)-\left[\varrho_{\mathrm{E}}^{*} \bar{C}\right](t, \mathrm{E})\right\}\right\}\right.$. 
THEOREM 9.2.

$$
c\left(X^{\prime}\right)-f^{*} c(X)=j_{*}\left\{-\frac{1}{\xi_{\mathrm{E}}} \varrho_{\mathrm{E}}^{*} c(Y)\left\{\left(1-\xi_{\mathrm{E}}\right)\left[\varrho_{\mathrm{E}}^{*} \bar{C}\right]\left(1+\xi_{\mathrm{E}}, \mathrm{E}\right)-\varrho_{\mathrm{E}}^{*} c(\mathrm{E})\right\}\right\} .
$$

Before proceeding to prove these results we shall explain the underlying concepts of the proof. There are two essential ideas involved. The basic one is to shift our attention, as explained in $\S 0$, from the blowing down morphism $f: X^{\prime} \rightarrow X$ to its associated "lifted correspondence " $\hat{f}$ (which is not a morphism) from $\hat{X}^{\prime}$ to $\hat{X}$ (the respective tangent direction bundles). The second idea is a purely technical trick. In order to avoid some very unpleasant, but not totally impractical, calculations we content ourselves with demonstrating that the left-hand side in Theorem 9.2, i.e. $\boldsymbol{c}\left(X^{\prime}\right)-f^{*} \boldsymbol{c}(X)$ is of the form $j_{*} w$, where $w$ is a class of $\mathcal{A}\left(Y^{\prime}\right)$ which depends on $Y$ and $\mathbf{E}$ only. In other words the varieties $X$ and $X^{\prime}$ are irrelevant: all that matters is the variety $Y$ and its normal bundle $\mathbf{E}$ in $X$. If therefore we replace $X$ by $\bar{E}$ (the projective closure of $\mathrm{E}$ ) we do not affect $w$, so that our theorems derive immediately from the results of the previous section.

First we explain, before proceeding to the detailed proofs, how we treat the correspondence $\hat{f}$. Let $\Delta^{\prime}$ (the prime is used to remind us that we are considering a blowing up of the diagonal $\Delta$ of $X \times X)$ be the graph on $X^{\prime} \times X$ of the morphism $f$ and let $\Gamma^{\prime}$ on $\hat{X}^{\prime} \times \hat{X}$ be the graph of the lifted correspondence $\hat{f}$. Let us denote the projections of $X^{\prime} \times X$ onto its components by $p_{x^{\prime}}, p_{x}$ and those of $\hat{X}^{\prime} \times \hat{X}$ by $p_{1}, p_{2}$. We shall also abbreviate $\varrho_{x^{\prime}}$ and $\varrho_{x}$ to $\varrho^{\prime}$ and $\varrho$ respectively (and later abbreviate $\xi_{X^{\prime}}, \xi_{x}$, the Grothendieck classes of the tangent direction bundles, to $\xi^{\prime}, \xi$ respectively) We summarise these notations in the diagram of fig. 9.2.

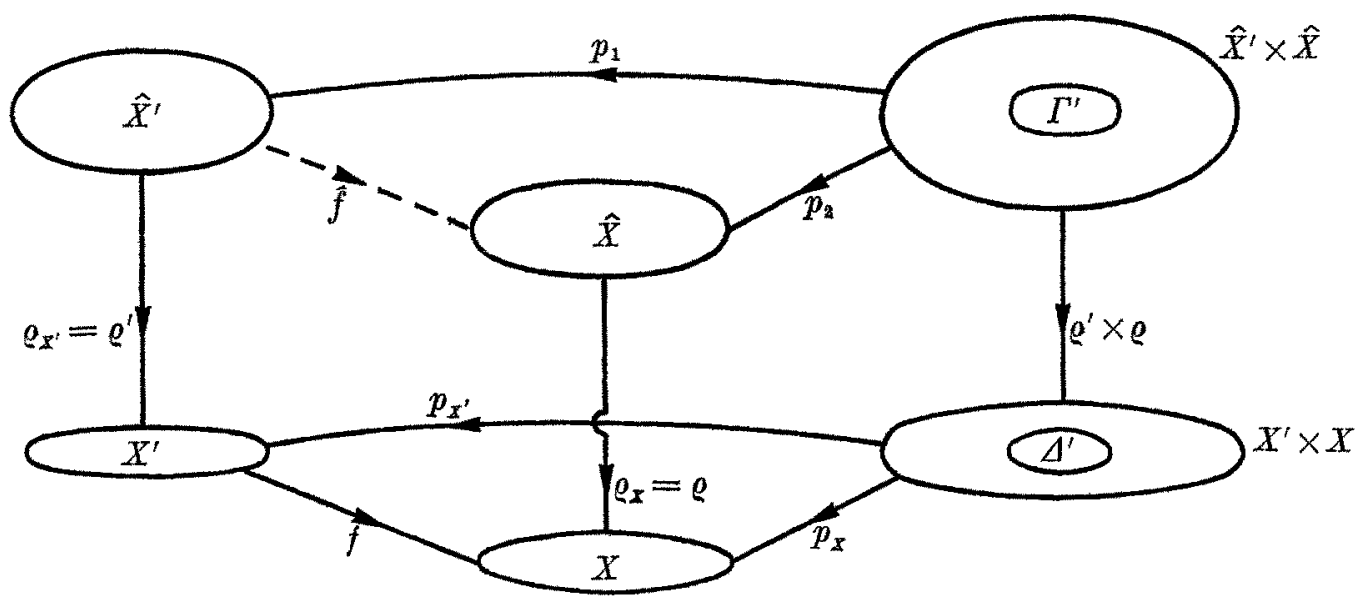

Fig. 9.2.

As $f$ is a birational morphism its graph $\Delta^{\prime}$ is isomorphic with $X^{\prime}$. But $\hat{f}$ is not an isomorphism. The tangent directions along the fibres of $Y^{\prime}$ are, as we shall see, fundamental. So $\Gamma^{\prime}$ is not isomorphic with $\hat{X}^{\prime}$ but, as could easily be checked in local co-ordinates as in PoRTEOUs [12] p. 121, with $\hat{\mathcal{X}}^{\prime}$ blown up along the subvariety 
$P\left(\mathrm{E}^{\prime}\right)$ (where $\mathrm{E}^{\prime}$, as previously, is the bundle of tangent vectors along the fibres of $P(E))$. In fact we don't consider $\Gamma^{\prime}$ directly, but we derive an isomorphic image of it by using the geometry of $\S 4$.

To see how the geometry of that section applies, we take $W=X^{\prime} \times X$, $\mathrm{E}_{1}=p_{x^{\prime}}^{*} \mathrm{~T}\left(X^{\prime}\right), \mathrm{E}_{3}=p_{X}^{*} \mathrm{~T}(X), Z=\hat{X}^{\prime} \times \hat{X}$ and consider fig. 4.1. Instead of looking direetly for $\Gamma^{\prime}$ on $Z$, we consider first its image under $\Phi^{-1}$ (the idea used in [15]). Now $\Phi^{-1}\left(\Gamma^{\prime}\right)$ is easily determined : it is simply $\hat{\Delta}^{\prime}$, the natural lift of $\Delta^{\prime}$ on $W$ to $\hat{W}=P(G)$ (cf. $[14,15])$ and $\hat{d}^{\prime}$ is isomorphic with $\hat{X}^{\prime}$. Let us denote by $h$ the isomorphism $h: \Delta^{\prime} \rightarrow X^{\prime}$ and by $\hat{h}$ the isomorphism $\hat{A}^{\prime} \rightarrow \hat{X}^{\prime}$, giving the diagram

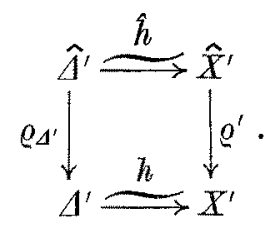

Now if we consider the proper transform by $\Psi^{-1}$ (still looking at fig. 4.1) of $\hat{\Delta}^{\prime}$ we shall get a variety $\Lambda$ on $N$. As $\Phi \Psi=\varrho_{s}$, the projection of $A$ to $Z$ will be the graph $\Gamma^{\prime}$ we require (and the projection is easily seen to be birational). Because the geometry on $H$ is richer than that on $Z$ it is better to discuss $A$ than $T^{\prime}$, and this we shall do.

So we have the following geometrical situation (fig. 9.4), which is simply fig. 4.1 adapted to our present situation.

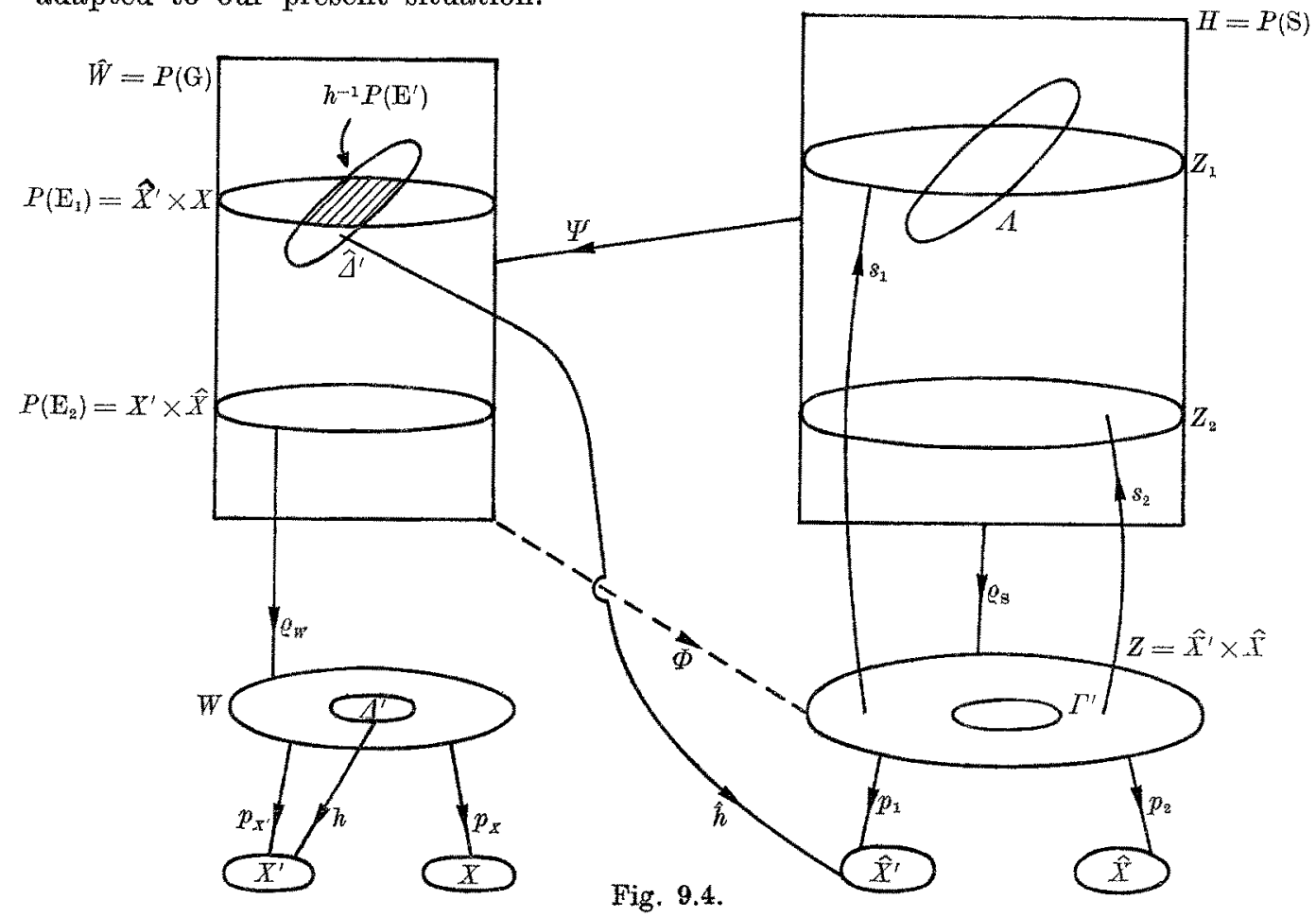


Let us now consider the intersections of $\lambda^{\prime}$ with the fundamental varieties of $\Psi^{-1}$. The intersection $\hat{X}^{\prime} \cap \hat{X}^{\prime} \times X$ consists of pairs $t_{x^{\prime}} \times x$ on $\hat{X}^{\prime} \times X$, where $t_{x^{\prime}}$ is a tangent direction to $X^{\prime}$ at $x^{\prime}, f x^{\prime}=x$ and the tangent direction at $x$ corresponding to $t_{x^{\prime}}$ under $\hat{f}$ is indeterminate. (If $t_{x^{\prime}}$ arises from a tangent vector $v_{x^{\prime}}$ to $X^{\prime}$, then the transform of $v_{x^{\prime}}$ by the tangent map $f_{T}$ is the zero vector.) This implies that $t_{x^{\prime}}$ is $a_{0}$ tangent direction to a fibre at a point of $P(\mathrm{E})$.

So

$$
\hat{\Delta}^{\prime} \cap \hat{X}^{\prime} \times X=\hat{h}^{-1} P\left(\mathrm{E}^{\prime}\right),
$$

where $\mathrm{E}^{\prime}$ is the bundle of tangents along the fibres of $P(\mathrm{E})$.

Similarly

$$
\hat{\Delta}^{\prime} \cap X^{\prime} \times \hat{X}=\emptyset
$$

because the tangent map $f_{n}: \mathrm{T}\left(X^{\prime}\right) \rightarrow \mathrm{T}(X)$ is a morphism.

The intersection of $\hat{A}^{\prime}$ and $\hat{X}^{\prime} \times X$ is of excessive dimension. If $n=\operatorname{dim} X$, $r=\operatorname{codim}_{x} \bar{Y}$, then

$$
\operatorname{dim} \hat{W}=4 n-1, \quad \operatorname{dim} \hat{\Delta}^{\prime}=2 n-1, \quad \operatorname{dim} \hat{X}^{\prime} \times X=3 n-1
$$

and $\operatorname{dim} P\left(\mathbf{E}^{\prime}\right)=\operatorname{dim} Y^{\prime}+r-1=n+r-2$. So the intersection has a dimension whose excess is $r-1$, which is certainly positive.

However if we apply $\Psi^{-1}$ the proper transform of $\hat{d}^{\prime}$ by $\Psi^{-1}$ is obtained by blowing up $\hat{d}^{\prime}$ along $\hat{h}^{-1} P\left(\mathbf{E}^{\prime}\right)$, which is isomorphie with $\hat{X}^{\prime}$ blown up along $P\left(\mathbf{E}^{\prime}\right)$. Let $A$ be the proper transform of $\hat{U}^{\prime}$ by $\Psi^{-1}$ and let $\lambda: A \hookrightarrow H$ be the inclusion mapping. We have the commutative diagram

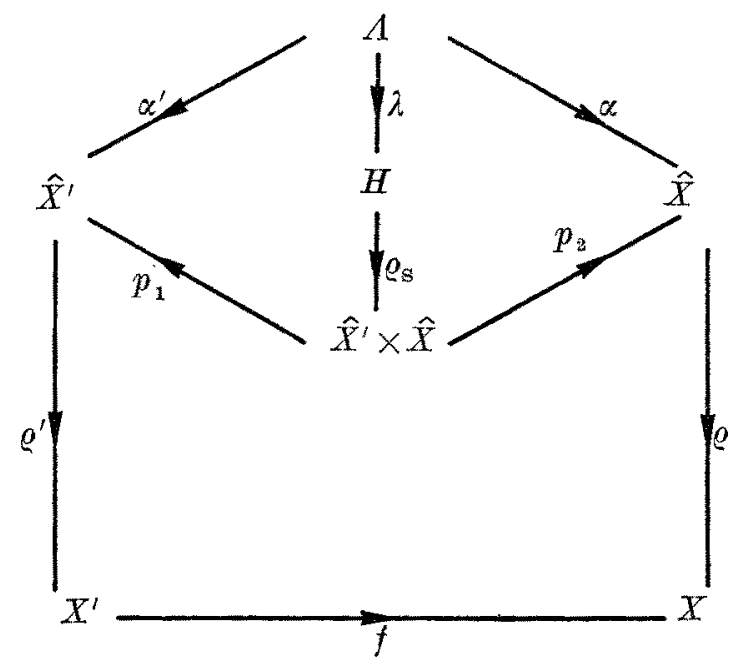

where $\varrho_{\mathrm{s}} \lambda$ is the projection of $A$ on $\Gamma^{\prime}$ and $\alpha^{\prime}, \alpha$ are simply $p_{1} \varrho_{\mathrm{s}} \lambda$ and $p_{2} \varrho_{\mathrm{s}} \lambda$ respectively. This means that $\alpha^{\prime}, \alpha$ are obtained by projecting $A$ onto the subvariety $\Gamma^{\prime}$ (the graph of $\hat{f}$ ) on $\hat{X}^{\prime} \times \hat{X}$ and then projecting onto the components of $\hat{X}^{\prime} \times \hat{X}$.

3-Annali di Matematica 
One can easily see that $\varrho_{\mathrm{s}} \lambda$ is an isomorphism. In fact $\varrho_{\mathrm{s}}^{-1} \Gamma^{\prime}$ is a bundle of projective lines over $\Gamma^{\prime}$ and $A$ gives a rational section $\Gamma^{\prime} \rightarrow e_{\mathrm{s}}^{-1} \Gamma^{\prime}$ which meets only one of the two sections induced by $s_{i}(i=1,2)$. Hence $\Lambda$ is a regular section and this shows that the map $\varrho_{\mathrm{s}} \lambda$ induced by the projection $\varrho_{\mathrm{s}}^{-1} \Gamma^{\prime} \rightarrow \Gamma^{\prime}$ is an isomorphism. We have also the commutative diagram

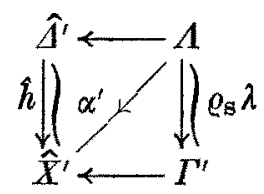

where $p_{1} \mid \Gamma^{\prime}: \Gamma^{\prime} \rightarrow \hat{X}^{\prime}$ is the blowing down morphism inverse to the blowing up of $\hat{X}^{\prime}$ along $P\left(\mathbf{E}^{\prime}\right)$. It follows that we have the blowing up diagram

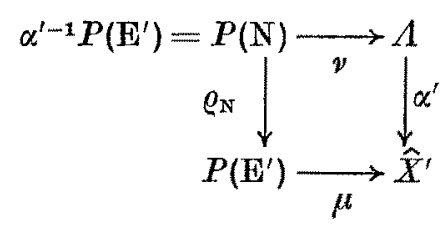

where $\mathrm{N}=\mathrm{N}\left(P\left(\mathrm{E}^{\prime}\right), \hat{X}^{\prime}\right)$ and $\mu, v$ are the inclusion maps.

Putting together the blowing up diagrams (9.1) and (9.8) with the diagram (9.7) we have the following composite diagram (9.9) which provides the key to what follows.

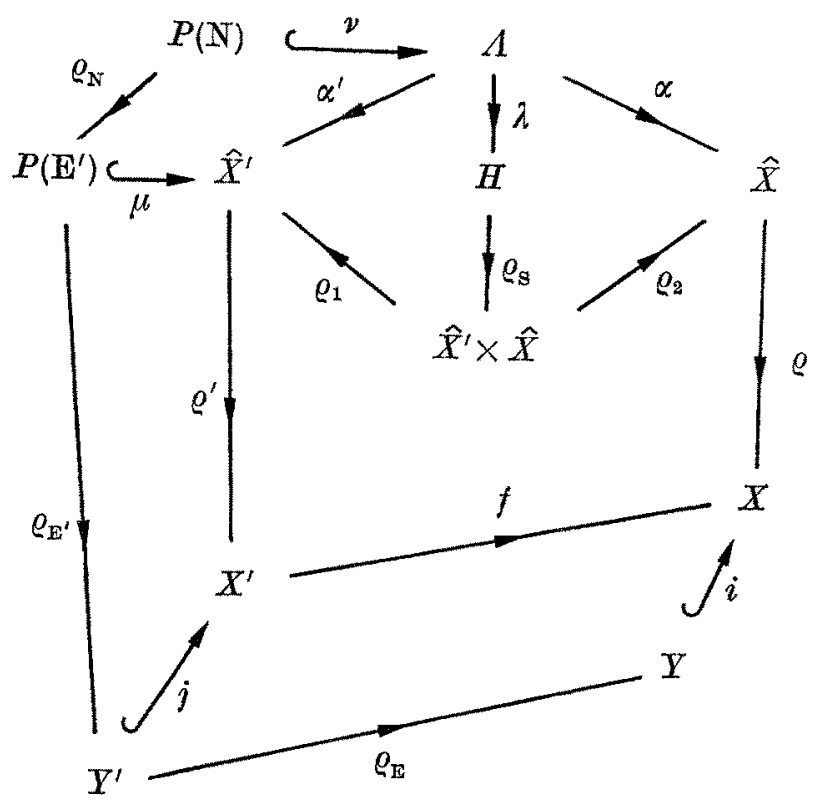

Now consider the intersection of $A$ and $Z_{1}$ in fig. (9.4). We know that the interference $A \cap Z_{1}$ is actually $P(\mathrm{~N})$ and we shall assume for the moment that

$$
A . Z_{1}=A \cap Z_{1}=P(\mathrm{~N}) .
$$


The justification of this assumption comes from a lemma on proper transforms of subvarieties in a blowing up which, to avoid interruption of our argument, we shall prove in an appendix $(\$ 10)$.

Now the class of $P(\mathrm{~N})$ on $A$ is $v_{*}(1)$, and that of $A . Z_{1}$ on $A$ is $\lambda^{*} \mathrm{cl}_{z} Z_{1}$. Now let us write, as suggested on p. $27, \xi_{x}=\xi, \xi_{x^{\prime}}=\xi^{\prime}$ and apply Theorem 4.1(i). We then get

$$
\nu_{*}(1)=\lambda^{*} \mathrm{cl}_{\mathrm{H}} Z_{1}=\lambda^{*}\left(\xi_{\mathrm{s}}-\varrho_{\mathrm{s}}^{*} p_{2}^{*} \xi\right)
$$

But as $\widehat{\Delta}^{\prime} \cdot \Psi\left(Z_{2}\right)=\emptyset($ by $(9.6))$ it follows that $A \cap Z_{2}=\emptyset$, so $\lambda^{*} \mathrm{cl}_{n} Z_{2}=0$. Applying now Theorem 4.1 (ii) we get

$$
\lambda^{*}\left(\xi_{\mathrm{s}}-\varrho_{\mathrm{s}}^{*} p_{1}^{*} \xi^{\prime}\right)=0
$$

Hence

$$
\nu_{*}(1)=\lambda^{*} \varrho_{\mathrm{s}}^{*} p_{1}^{*} \xi^{\prime}-\lambda^{*} \varrho_{\mathrm{s}}^{*} p_{2}^{*} \xi=\alpha^{*} \xi^{\prime}-\alpha^{*} \xi\left(^{\dagger}\right) .
$$

Now using the formula (3.10) we get

$$
\left[\varrho^{\prime *} \bar{C}\right]\left(\xi^{\prime}, X^{\prime}\right)=0 \text { and } \quad\left[\varrho^{*} \bar{C}\right](\xi, X)=0
$$

From (9.14) we deduce

$$
0=\alpha^{*}\left[\varrho^{*} \bar{C}\right](\xi, X)=\left[\alpha^{*} \varrho^{*} \bar{C}\right]\left(\alpha^{*} \xi, X\right)
$$

So applying (9.13) we get

$$
\left[\alpha^{*} \varrho^{*} \bar{C}\right]\left(\alpha^{*} \xi^{\prime}-\nu_{*}(1), X\right)=0
$$

which we can write in the form

$$
\left[\alpha^{*} \varrho^{*} \bar{C}\right]\left(\alpha^{\prime *} \xi^{\prime}, X\right)+\nu_{*}(1) \cdot u=0
$$

where

$$
u=\frac{1}{v_{*}(1)}\left\{\left[\alpha^{*} \varrho^{*} \bar{C}\right]\left(\alpha^{\prime *} \xi^{\prime}-v_{*}(1), X\right)-\left[\alpha^{*} \varrho^{*} \bar{C}\right]\left(\alpha^{\prime *} \xi^{\prime}, X\right)\right\}
$$

(†) The vital formula $\nu^{*}(1)=\alpha^{\prime} \xi^{\prime}-\alpha^{*} \xi$ can be obtained in other ways. We first found it by considering the Grothendieck classes $\xi^{\prime}$ and $\xi$ as defined by "invariant lifts " (of. [10]), using a pencil of primals on $X$ not specially related to $Y$ and the images of this pencil by $f^{-1}$ on $X^{\prime}$. Porteous has an alternative suggestion which, like our original idea, is not easier to present than the one given here. 
But, by the projection formula (1.1),

$$
\nu_{*}(1) \cdot u=\nu_{*}\left(\nu^{*} u\right)
$$

Also, by (3.7) and lemma (3.5)

$$
v^{*} v_{*}(1)=t_{1}(P(\mathrm{~N}), A)=c_{1}\left(\check{\mathrm{L}}_{\mathrm{N}}\right)=-\xi_{\mathrm{N}} .
$$

Again, using (9.9),

$$
\nu^{*} \alpha^{\prime *} \xi^{\prime}=\varrho_{N}^{*} \mu^{*} \xi^{\prime}=\varrho_{\mathrm{N}}^{*} \xi_{\mathrm{E}^{\prime}}
$$

So

$$
v^{*} u=-\frac{1}{\xi_{\mathrm{N}}}\left\{\left[v^{*} \alpha^{*} \varrho^{*} \bar{C}\right]\left(\varrho_{\mathrm{N}}^{*} \xi_{\mathrm{E}^{\prime}}+\xi_{\mathrm{X}}, X\right)-\left[v^{*} \alpha^{*} \varrho^{*} \bar{C}\right]\left(\varrho_{\mathrm{N}}^{*} \xi_{\mathrm{E}^{\prime}}, X\right)\right\}
$$

But, again using (9.9), $v^{*} \alpha^{*} \varrho^{*}=\varrho_{X}^{*} \varrho_{\mathrm{E}^{\prime}}^{*} \varrho_{\mathrm{E}}^{*} i^{*}$, and also (since $\left.\mathrm{E}=N(Y, X)\right)\left[i^{*} \bar{C}\right](t, X)=$ $=\bar{C}(t, Y) \bar{C}(t, \mathbf{E})$.

Thus $v^{*} u$ is a polynomial of the form

$$
v^{*} u=\Sigma\left(\varrho_{N}^{*} a_{i}\right) \xi_{N}^{i},
$$

where $a_{i} \in \mathcal{A} P\left(\mathrm{E}^{\prime}\right)$ (but $a_{i}$ is not necessarily of co-dimension $i$ ) and $a_{i}$ depends only on $\mathbf{E}$ and $Y$. We then have

Lemrar 9.4. - The class $\varrho_{\mathbb{N} *} \nu^{*} u$ depends only on $\mathrm{E}, Y$.

This follows from (9.17) because, by the projection formula,

$$
\varrho_{\mathrm{N}}\left(\left(\varrho_{\mathrm{N}}^{*} a_{i}\right) \xi_{\mathrm{N}}^{*}\right)=a_{i} \varrho_{\mathrm{N}}\left(\xi_{\mathrm{N}}^{i}\right)
$$

where $\varrho_{\mathrm{N}^{*}}\left(\xi_{\mathrm{N}}^{i}\right)$ are the Segre classes of $\mathrm{N}$ which are determined by the Chern classes of $\mathrm{N}$. Next we show that these Chern classes of $\mathrm{N}$ also depend only on $\mathrm{E}, Y$. Indeed, if we apply Theorem 7.3 with $i: V \rightarrow W$ replaced by $j: Y^{\prime} \rightarrow X^{\prime}$, and $\mathrm{E}$, E replaced by $T_{X^{\prime}}, E^{\prime}$ respectively, we obtain

$$
0 \rightarrow \varrho_{\mathrm{E}^{\prime}}^{*}\left(j^{*} \mathrm{~T}_{\mathbf{X}^{\prime}} / \mathbf{E}^{\prime}\right) \otimes \mathrm{L}_{\mathrm{E}^{\prime}} \rightarrow \mathrm{N} \rightarrow \varrho_{\mathrm{E}^{\prime}}^{*} \check{L}_{\mathrm{E}} \rightarrow 0
$$

We also have the commutative diagram

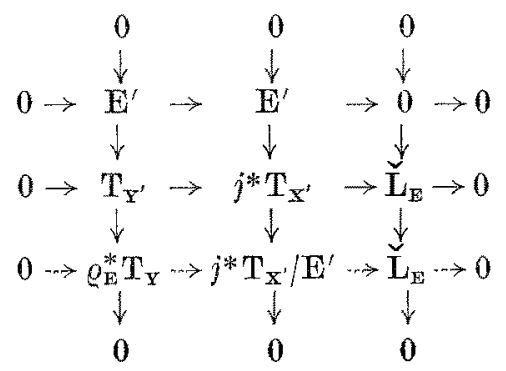


It follows that $C(t, \mathrm{~N})$ can be calculated from (9.21) in terms of $\mathrm{E}$ and $Y$ only. Now since, by $(9.9) \alpha^{*} \varrho^{*}=\alpha^{\prime *} \varrho^{\prime *} f^{*}$, we deduce from (9.16) with the aid of $(9.18)$

$$
\left[\alpha^{*} \varrho^{*} f^{*} \bar{C}\right]\left(\alpha^{*} \xi^{\prime}, X\right)+v_{*} v^{*} u=0 .
$$

Next apply $\alpha_{*}^{\prime}$ to $(9.22)$, remembering that $\alpha_{*}^{\prime} \alpha^{\prime *}$ is the identity (because $\alpha^{\prime}$ is a birational morphism) and since $\alpha_{*}^{\prime} \nu_{*}=\mu_{*} Q_{\mathrm{N}^{*}}$ we get

$$
\left[\mathfrak{Q}^{\prime *} f^{*} \bar{C}\right]\left(\xi^{\prime}, \bar{X}\right)+\mu_{*} \varrho_{\mathrm{N}} \nu^{*} u=0,
$$

or

$$
\left[\varrho^{\prime *} f^{*} \bar{C}\right]\left(\xi^{\prime}, X\right)+\mu_{*} v=0
$$

where, by lemma 9.4, $v$ depends only on $Y$, E. Hence using (9.14) we now have

$$
\left[\varrho^{\prime * \bar{C}}\right]\left(\xi^{\prime}, X^{\prime}\right)-\left[\varrho^{\prime *} f^{*} \bar{C}\right]\left(\xi^{\prime}, X\right)=\mu_{*} v,
$$

or

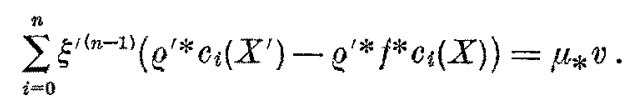

If we apply Theorem 3.8 , in which we now have $\bar{a}=\boldsymbol{c}\left(X^{\prime}\right)-f^{*} \boldsymbol{c}(X)$, we obtain

$$
\boldsymbol{c}\left(X^{\prime}\right)-f^{*} \boldsymbol{c}(X)=\varrho_{*}^{\prime}\left(\mu_{*} v \cdot \sum_{0}^{\infty} \xi^{\prime i}\right) \cdot \boldsymbol{c}\left(X^{\prime}\right)
$$

But, by the projection formula (1.1),

$$
\mu_{*} v: \sum_{\mathbf{0}}^{\infty} \xi^{i}=\mu_{*}\left(v, \mu^{*} \sum_{0}^{\infty} \xi^{\prime^{i}}\right)=\mu_{*}\left(v \sum_{0}^{\infty} \xi_{\mathrm{E}^{\prime}}^{i}\right)
$$

Substituting this in $(9.24)$ we get

$$
\begin{aligned}
\boldsymbol{c}\left(X^{\prime}\right)-f^{*} \boldsymbol{c}(X) & =\varrho_{*}^{i} \mu_{*}\left(v \sum_{0}^{\infty} \xi_{\mathrm{E}^{\prime}}^{i}\right) \cdot \boldsymbol{c}\left(X^{\prime}\right) \\
& =j_{*} \varrho_{\mathrm{E}^{\prime} *}\left(v \sum_{0}^{\infty} \xi_{\mathrm{E}^{\prime}}^{i}\right) \cdot \boldsymbol{c}\left(X^{\prime}\right) \\
& =j_{*}\left(\varrho_{\mathrm{E}^{\prime}}\left(v \sum_{0}^{\infty} \xi_{\mathrm{E}^{\prime}}^{i}\right) \cdot j^{*} \boldsymbol{c}\left(X^{\prime}\right)\right) .
\end{aligned}
$$

But since $\mathrm{N}\left(Y^{\prime}, X^{\prime}\right)=\breve{\mathrm{L}}_{\mathrm{E}}$ (lemma 3.5) it follows, by (3.1), that

$$
j^{*} c\left(X^{\prime}\right)=\left(1-\xi_{\mathrm{E}}\right) c\left(Y^{\prime}\right) .
$$


And as $Y^{\prime}=P(\mathbf{E})$ it follows, by theorem 7.2, that $c\left(Y^{\prime}\right)$ depends on $Y$ and $\mathbf{E}$. So we have finally established that

$$
\boldsymbol{c}\left(X^{\prime}\right)-f^{*} \boldsymbol{c}(X)=j_{*} w
$$

where $w$ depends on $\mathrm{E}$ and $Y$ only. Or equivalently

$$
\bar{C}\left(t, X^{\prime}\right)-f^{*} \bar{C}(t, X)=j_{*} W(t)
$$

where $W(t) \in A\left(Y^{\prime}\right)[t]$ and its coefficients depend on $Y, \mathrm{E}$ only.

Given the variety $Y$ and the bundle $\mathrm{E}$ we can consider the bundle $P(\mathrm{G})=P\left(\mathrm{E} \oplus \mathbf{1}_{Y}\right)$, the projective completion of $\mathbf{E}$. Let us now adopt the notation of $\$ 8$ except that objects oceurring there (other than $\mathrm{E}, Y, U^{\prime}$ ) which are different from ones with the same name considered in this section will be denoted by a tilde. We shall, for example, speak of $\tilde{X}=P(G), \tilde{H}=\tilde{X}^{\prime}, \tilde{i}, \tilde{j}, \tilde{S}, \tilde{Z}_{1}, \tilde{Z}_{2}$ and we shall also write $\Psi=\tilde{f}$.

Then, applying the results we have just obtained to the blowing up diagram

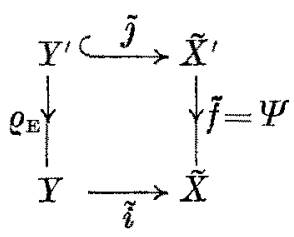

we get $\bar{C}\left(t, \tilde{X}^{\prime}\right)-[\Psi * \bar{C}](t, \tilde{X})=\tilde{\jmath}_{*} W(t)$.

But by Theorem 8.1 we deduce

$$
\tilde{j}_{*} W(t)=\tilde{j}_{*}\left\{-\frac{1}{\xi_{\mathrm{E}}}\left[\varrho_{\mathrm{E}}^{*} \bar{C}\right](t, X)\left\{\left(1-\xi_{\mathrm{E}} \mid t\right)\left[\varrho_{\mathrm{E}}^{*} \bar{C}\right]\left(t+\xi_{\mathrm{E}}, \mathrm{E}\right)-\left[\varrho_{\mathrm{E}}^{*} \bar{C}\right](t, \mathrm{E})\right\}\right\}
$$

But, by $(8.1), \varrho_{\tilde{\mathbb{S}}} \tilde{j}=\mathrm{Id}_{\boldsymbol{Y}^{\prime}}$, so $\varrho_{\tilde{\mathbb{S}}^{*}} \tilde{j}_{*}=\mathrm{Id}$ on $A\left(Y^{\prime}\right)$. If we then apply $\varrho_{\tilde{s}^{*}}$ to each side of $(9.25)$ we effectively cancel the $\tilde{\jmath}_{*}$ on each side and so Theorem (9.1) follows at once.

\section{0. - Appendix. A lemma on proper transforms.}

Suppose we have the blowing up diagram (9.1). We wish to consider the proper transforms of subvarieties of $X$ having suitable intersections with $Y$. We have to distinguish the interference $A \cap B$ of two subvarieties from the intersection cycle (where it is defined) $A . B$. The object of the following lemma is to give a sufficient condition for the two to coincide. So we wish to establish the following

LEMMa 10.1. - Suppose $U$ is a non-singular subvariety of $X$ sueh that $U \nsubseteq Y$ and $V=U \cap Y$ is irredueible and non-singular. Let $U^{\prime}$ (on $X^{\prime}$ ) be the proper transform 
of $D$ by $f^{-1}$ and denote by $g$ the mapping $f \mid U^{\prime}$. Suppose further that $g^{-1}$ is the blowing up of $V$ along $V$, so that $V^{\prime}=g^{-1} V=U^{\prime} \cap Y^{\prime}$ and is irreducible. Under all these conditions the intersection $U^{\prime} . Y^{\prime}$ is defined and is equal to $\nabla^{\prime}$.

Let $\mathrm{E}=\mathrm{N}(Y, X), \mathrm{F}=\mathrm{N}(V, U)$. Then $Y^{\prime}=P(\mathrm{E}), V^{\prime}=P(\mathrm{~F}), \check{\mathrm{L}}_{\mathrm{E}}=\mathrm{N}\left(X^{\prime}, X^{\prime}\right)$, $\check{\mathrm{L}}_{\mathrm{F}}=\mathrm{N}\left(\nabla^{\prime}, U^{\prime}\right)$. As $\mathrm{F}$ is contained in $\mathrm{E}, \check{\mathrm{L}}_{\mathrm{F}}$ is induced on $V^{\prime}$ by $\check{\mathrm{L}}_{\mathrm{E}}$.

Now $Y^{\prime}$ is a primal of $X^{\prime}, V^{\prime}=U^{\prime} \cap Y^{\prime}$ and, by hypothesis, $\operatorname{codim}_{\sigma^{\prime}} V^{\prime}=1$, so the intersection $U^{\prime} . Y^{\prime}$ is defined in $X^{\prime}$. As $V^{\prime}$ is irreducible we need only prove that its multiplicity is one in the intersection $V^{\prime} . Y^{\prime}$. Hence it is enough to show that at any point $z$ on $V^{\prime}, \mathbf{T}\left(U^{\prime}\right)_{z}$ and $\mathbf{T}\left(Y^{\prime}\right)_{z}$ are transversal in $\mathrm{T}\left(X^{\prime}\right)_{z}$. As $\mathbf{T}\left(Y^{\prime}\right)_{z}$ is a prime in $\mathbf{T}\left(X^{\prime}\right)_{z}$, it suffices to show that $\mathbf{T}\left(U^{\prime}\right)_{z} \nsubseteq \mathbf{T}\left(Y^{\prime}\right)_{z}$. This, however, is an immediate consequence of the following commutative diagram (obtained by pulling back to $z$ the normal bundle sequences of the form (3.1) for $V^{\prime} \subset U^{\prime}$ and $Y^{\prime} \subset X^{\prime}$.

$$
\begin{gathered}
0 \rightarrow \mathrm{T}\left(V^{\prime}\right)_{z} \rightarrow \mathrm{T}\left(U^{\prime}\right)_{z} \rightarrow\left(\check{\mathrm{L}}_{\bar{F}}\right)_{z} \rightarrow 0 \\
\downarrow \\
\downarrow \\
0 \rightarrow \mathrm{T}\left(Y^{\prime}\right)_{z} \rightarrow \mathrm{T}\left(X^{\prime}\right)_{z} \rightarrow\left(\check{\mathrm{L}}_{\varepsilon}\right)_{z} \rightarrow 0,
\end{gathered}
$$

since the image of $\mathrm{T}\left(Y^{\prime}\right)_{z}$ in $\left(\check{\mathrm{L}}_{\mathrm{E}}\right)_{z}$ is zero and that of $\mathrm{T}\left(U^{\prime}\right)_{z}$ in $\left(\breve{\mathrm{L}}_{\mathrm{F}}\right)_{z}$ is not.

This establishes the lemma.

Note that if we blow up $X$ additionally along a variety $Y_{1}$ disjoint from both $Y$ and $U$, the situation is unaffected.

For the application of this lemma needed in $\$ 9$ we have to replace $X$ by $\hat{W}=\widehat{X^{\prime} \times X}$ and $U$ by $\hat{A}^{\prime}$. The variety $\hat{W}$ is blown up along $\hat{X}^{\prime} \times X$ (which replaces $Y$ ) and $X^{\prime} \times \hat{X}$ which, since it is disjoint from both $\hat{X}^{\prime} \times X$ and $\hat{A}^{\prime}$ (which correspond to $\bar{Y}, U$ in the lemma) can be taken as $\underline{V}_{1}$. The conditions of the lemma are satisfied with $\bar{Y}^{\prime}=Z_{1}$, and $U^{\prime}=\Lambda$, so we can assert, as we did, that $\Lambda . Z_{1}=\Lambda \cap Z_{1}$.

\section{REFERENCES}

[1] A. BoREL - F. HIRZEBRUCH, Characteristic classes and homogeneous spaces, I, II, III, Am. Journal of Math., 80 (1958), pp. 458-538; 81 (1959), pp. 315-382; 82 (1960), pp. $491-504$.

[2] A. Bofel - J.-P. Serre, Le théoreme de Riemann-Roch, Bull. Soc. Math. de France, 86 (1958), pp. 97-136.

[3] N. BourBakI, Eléments de Mathematique, vol. XXXIII and XXXVI.

[4] Seminaire C. Chevalley, Anneaux de Chow et applications, Secretariat mathematique, 11 Rue Pierre Curie, Paris 5e, 1958.

[5] Gh. Galbura, Sui covarianti di immersione, Rend. di Mat., 25 (1966), pp. 239-247 (Castelnuovo centenary volume).

[6] Gh. Galbura - A. T. Lascu, Eclatement des olasses de Chern d'une variété algébrique, Rev. Rom. Math. Pures et Appl., 12 (1967), pp. 1255-1258.

[7] A. Grothendieck, La théorie des classes de Chern, Bull. Soc. Math. de France, 86 (1958), pp. $137-159$. 
[8] F. HirzerRuch, Topological methods in algebraic geometry, Grundlehren der Math. Wissenschaften, 131 (1966).

[9] S. A. Ilon - A. W. Ingleton - A. T. LAscu, On a formula of D. B. Scoth (in preparation) (to appear in J. London Math. Soc.).

[10] A. W. IngLeton - D. B. ScotT, The tangent direction bundle of an algebraic variety and generalized Jacobians of linear systems, Annali di Mat., (4), 56 (1961), pp. 359-374.

[11] J.-P. Jovanolov, Riemann.Roch sans dénominateurs, Inventiones Math., 11 (1970), pp. 15-26.

[12] I. R. Porreovs, Blowing up Chern classes, Proo, Cambridge Phil. Soc., 56 (1960), pp. 118-124.

[13] I. R. Pokreous, Simple Singularities of Maps, in Proc. Liverpool Singularities Symposium I, Springer Lecture Notes in Mathematics, vol. 192.

[14] D. B. ScotT, Natural lifts and the covariant systems of Todd, J. London Math. Soc., (2), 1 (1969), pp. 709-718.

[15] D. B. Scotr, A topological approach to branch and double curves of correspondences between algebraic surfaces, Convegno Internazionale Enriques, Milano, 1971.

[16] B. SEGRE, Nuovi metodi e risultati nella geometria sulle varietà algebriche, Annali di Mat., (4), 35 (1953), pp. 1-127.

[17] B. SEGRe, Dilatazioni e varietà canoniche sulle varietà algebriche, Annali di Mat., (4), 37 (1954), pp. 139-155.

[18] J. A. TodD, Birational transformations with isolated fundamental points, Proc. Edinburgh Math. Soc., (2), 5 (1938), pp. 117-124.

[19] J. A. ToDd, Birational transformations possessing fundamental curves, Proc. Cambridge Phil. Soc., 34 (1938), pp. 144-155.

[20] J. A. Topd, Invariant and covariant systems on an algebraie variety, Proc. London Math. Soc., (2), 46 (1940), pp. 199-230.

[21] J. A. Tovd, Birational transformations with a fundamental surface, Proc. London Math. Soc., (2), 47 (1941), pp. 81-100.

[22] J. A. Tond, Canonical systems on algebraic varieties, Boletin de la Sociedad Matematica Mexicana (1957), pp. 38-44.

[23] A. J. H. M. VAN DE VEN, Characteristic classes and monoidal transformations, Indag. Math., 18 (1956), pp. 571-578. 